\title{
Norm-Conserving Pseudopotentials and Basis Sets to Explore Actinide Chemistry in Complex Environments
}

\author{
Jun-Bo Lu ${ }^{\mathrm{a}, \mathrm{c}}$, David C. Cantu ${ }^{\mathrm{b}}$, Cong-Qiao Xuc ${ }^{\mathrm{c}}$, Manh-Thuong Nguyen ${ }^{\mathrm{d}}$, Han-Shi Hu ${ }^{\mathrm{a}}$, \\ Vassiliki-Alexandra Glezakou ${ }^{\mathrm{d}^{*}}$, Roger Rousseau ${ }^{\mathrm{d} *}, \mathrm{Jun} \mathrm{Li}^{\mathrm{a}, \mathrm{c}^{*}}$
}

${ }^{a}$ Department of Chemistry and Key Laboratory of Organic Optoelectronics \& Molecular Engineering of the Ministry of Education, Tsinghua University, Beijing 100084, China

${ }^{\mathrm{b}}$ Chemical and Materials Engineering, University of Nevada, Reno, Reno, Nevada 89557, United States

${ }^{c}$ Department of Chemistry, Southern University of Science and Technology, Shenzhen 518055, China

d Basic and Applied Molecular Foundations, Pacific Northwest National Laboratory, P.O. Box 999, Richland, Washington 99352, United States

*Corresponding authors: vanda.glezakou@pnnl.gov, roger.rousseau@pnnl.gov, junli@tsinghua.edu.cn 


\begin{abstract}
We have developed a new set of norm-conserving pseudopotentials and companion Gaussian basis sets for the actinide (An) series (Ac - Lr) using the Goedecker, Teter and Hutter (GTH) formalism with the Perdew, Burke and Ernzerhof (PBE) exchange-correlation functional of generalized gradient approximation (GGA). To test the accuracy and reliability of the newly parameterized An-GTH pseudopotentials and basis sets, a variety of benchmarks on actinide-containing molecules are carried out and compared to all-electron and available experimental results. The new pseudopotentials include both medium- $\left([\mathrm{Xe}] 4 \mathrm{f}^{14}\right)$ and large-core $\left([\mathrm{Xe}] 4 \mathrm{f}^{14} 5 \mathrm{~d}^{10}\right)$ options that have successfully reproduced structures and energetics, particularly redox processes. The medium-core size set, in particular, reproduce all-electron calculations over multiple oxidation states from 0 to VII, whereas the large-core set is suitable only for the early series elements and low oxidation states. The underlying reason for these transferability issues are discussed in detail. This work fills a critical void in the literature for studying the chemistry of $5 f$-block elements in condensed phase.
\end{abstract}




\section{INTRODUCTION}

Since their inclusion in the periodic table of elements by Seaborg, actinides have played a paramount role in science and human society. ${ }^{1-2}$ Besides the applications in energy resource and industry, the potential usefulness of actinide-containing compounds has been extended in catalysis ${ }^{3-6}$, single

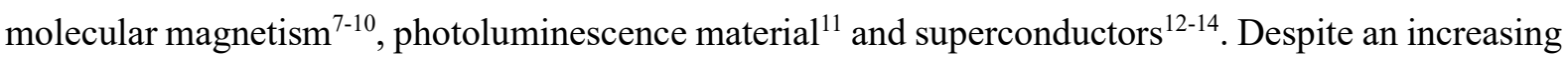
attention on actinide chemistry, large-scale condensed phase electronic structure simulations of actinide-containing systems are lacking, due in large part to challenges on description of the huge number of electrons, especially the $5 f$-state electrons, as well as strong relativistic and electron correlation effects. The use of all-electron calculations with basis sets that consider relativistic effects will always be required to predict some actinide properties, e.g., nuclear magnetic resonance. ${ }^{15-20}$ However, the use of accurate pseudopotentials, that appropriately replace atomic core electrons and account for scalar relativistic effects, has been proven to be an effective treatment to model actinides with considerably reduced computational cost. ${ }^{21-23}$ Computational modeling and simulations of actinide systems in the condensed phase are particularly challenging as pseudopotentials and companion basis sets have to be constructed to reduce the computational cost and achieve linear scaling for actinides in bulk solids, surfaces and solutions with full explicit solvent boxes. ${ }^{24}$

Large-scale computational modeling of actinide-containing systems is possible with density functional theory (DFT), which requires reliable pseudopotentials and basis sets that can accurately model the electronic structure and replicate reactivity to a level of fidelity comparable to quantum chemistry wavefunction approaches. Currently, efficient and accurate actinide pseudopotentials and basis sets, for ab initio molecular dynamics (AIMD) simulations with actinide-containing systems, are completely lacking due to the difficulty of describing the $5 f$-states. Accurate shape-consistent, energyconsistent, and norm-conserving pseudopotentials exist for actinides, and they are already commonly used in molecular quantum chemistry community, as shown in Table $1 .{ }^{21-23,25-34}$ However, applications of them are limited in practice when performing large-scale AIMD simulations in condensed phase, due to the cubic scaling arising from nonlocal part of these pseudopotentials. This obstacle can be circumvented via the introduction of separable form pseudopotentials. ${ }^{35}$ 
Table 1. Previous published pseudopotentials for actinide elements.

\begin{tabular}{|c|c|c|c|c|c|}
\hline Name & Type & Elements & Year & Core Elec. ${ }^{c}$ & Ref \\
\hline \multirow{3}{*}{ LANL2DZ ECP } & \multirow{3}{*}{ Shape-consistent } & $\mathrm{U}$ & 1979 & 78 & 21 \\
\hline & & $\mathrm{Th}$ & 1981 & 78 & 22 \\
\hline & & $\mathrm{Pu}$ & 1987 & 78 & 23 \\
\hline \multirow{2}{*}{ CRENBL ECP } & \multirow{2}{*}{ Shape-consistent } & Ac-Am & 1991 & 78 & 25 \\
\hline & & $\mathrm{Cm}-\mathrm{Lr}$ & 1991 & 60 & 25 \\
\hline ЕСР60MHF & & Ac-Lr & 1993 & 60 & 26 \\
\hline ECP60MWB & & Ac-Lr & 1994 & 60 & 27 \\
\hline ECP78MWB & Energy-consistent & Ac-U & 2008 & 78 & 28 \\
\hline \multirow{2}{*}{ ECP60MDF } & & $\mathrm{U}$ & 2009 & 60 & 29 \\
\hline & & Ac-Pa & 2014 & 60 & 30 \\
\hline \multirow{2}{*}{$\mathrm{T}-\mathrm{M} \mathrm{PP}^{\mathrm{a}}$} & \multirow{2}{*}{ Norm-conserving } & $\mathrm{U}$ & 2006 & 78 & 31 \\
\hline & & $\mathrm{Pu}$ & 2013 & 78 & 32 \\
\hline \multirow{2}{*}{ GTH PP } & \multirow{2}{*}{ Norm-conserving } & $\mathrm{U}$ & 2013 & 78 & 33 \\
\hline & & $\mathrm{U}$ & 2018 & 78 & 34 \\
\hline
\end{tabular}

a $\mathrm{T}-\mathrm{M} P \mathrm{P}=$ Troullier-Martin pseudopotential.

${ }^{\mathrm{b}}$ GTH PP $=$ Geodecker, Teter and Hutter pseudopotential.

${ }^{\mathrm{c}}$ Core Elec. means electron numbers of core region.

The Godecker, Teter and Hutter (GTH)-type formalism produces separable, Gaussian-type, normconserving pseudopotentials, that are sufficiently flexible in their functional form to allow for transferability over many chemically-relevant charge states. ${ }^{36-38}$ The utilization of GTH pseudopotentials with a mixed Gaussian-planewave (GPW) scheme is a highly effective and efficient way to perform linear scaling AIMD simulations ${ }^{39}$ To date, there are just two published large-core GTH pseudopotentials for uranium, but none are available for the rest of the actinide series..$^{33,34}$

In this work, we fill this critical gap by providing a complete set of well-tested pseudopotentials and companion basis sets capable of reproducing the redox chemistry of the entire actinide series. Recently, we successfully optimized GTH-type pseudopotentials and accompanying basis sets for the 
lanthanides, ${ }^{40-41}$ and their accuracy was illustrated by a series of molecular and solid-state electronic structure calculations. ${ }^{41}$ Considering the similarities between lanthanides and actinides, the optimization scheme applied for lanthanides could be used to optimize GTH pseudopotentials and basis sets for actinides. The transferability of actinide pseudopotentials is dictated by oxidation states and spatial correlation between their $5 f$ and $5 d$ orbitals. Given the breadth of actinide oxidation states, we developed both large- and medium-core GTH pseudopotentials, for all the actinide elements. Associated Gaussian basis sets, using the molecularly optimized (MOLOPT) formalism, were prepared for both sets of pseudopotentials. ${ }^{42}$ The efficacy of our actinide pseudopotentials and basis sets is demonstrated by a series of molecular benchmarks compared to both all-electron calculations as well as experimental measurements. Our systematically developed actinide pseudopotentials and basis sets will enable largescale DFT calculations and AIMD simulations of actinide-containing systems in the condensed phase, and can serve as a starting point for pseudopotentials for more accurate methods like hybrid DFT functionals or GW approaches.

The paper is organized as follows. In section 2, the theories of GTH pseudopotentials and basis sets and optimization scheme for them are shown. In section 3, transferability tests and molecular benchmarks are presented, validating the performance. Finally, conclusions are summarized in section 4.

\section{THEORETICAL AND COMPUTATIONAL METHODOLOGY}

\subsection{GTH pseudopotentials and MOLOPT basis sets}

The norm-conserving, separable, dual-space GTH pseudopotentials consist of a local and a nonlocal part. The local part is described by the following equation:

$$
V_{l o c}^{P P}(r)=-\frac{z_{i o n}}{r} \operatorname{erf}\left(\alpha^{P P} r\right)+\sum_{i}^{4} C_{i}^{P P}\left(\sqrt{2} \alpha^{P P} r\right)^{2 i-2} \times \exp \left[-\left(\alpha^{P P} r\right)^{2}\right]
$$

where

$$
\alpha^{P P}=\frac{1}{\sqrt{2} r_{l o c}^{P P}}
$$

where erf denotes the error function, $r_{l o c}$ is the range of the Gaussian charge distribution, and $Z_{i o n}$ is the ionic charge.

The non-local part is formulated by 


$$
V_{n l}^{P P}\left(\boldsymbol{r}, \boldsymbol{r}^{\prime}\right)=\sum_{l m} \sum_{i j}<\boldsymbol{r}\left|p_{i}^{l m}>h_{i j}^{l}<p_{j}^{l m}\right| \boldsymbol{r}^{\prime}>
$$

where $h$ is scattering matrix, and $\left\langle\boldsymbol{r} \mid p_{i}^{l m}\right\rangle$ is Gaussian-type projectors

$$
<\boldsymbol{r} \mid p_{i}^{l m}>=N_{i}^{l} Y^{l m}(r) r^{l+2 i-2} \exp \left[-\frac{1}{2}\left(\frac{r}{r_{l}}\right)^{2}\right]
$$

where $N_{i}^{l}$ denotes a normalization constant and $Y^{l m}$ denotes a Laplace spherical harmonic.

GTH pseudopotentials are explicitly separable, with the trade-off that one needs to fit the components of a scattering matrix elements $h_{i j}$. The parameters $r_{l o c}, C_{i}$ in local part, and $r_{l}$ and $h_{i j}$ in nonlocal part, in equations 1-4 are fitted in our optimizations. For the companion basis sets, we adopted the molecularly optimized (MOLOPT) Gaussian basis sets as proposed by VandeVondele and Hutter. ${ }^{42}$ The $s, p, d, f$ and $g$ states will share the same Gaussian exponents in the current MOLOPT basis set scheme for the sake of efficiency.

The general procedure to optimize the actinide GTH pseudopotentials and corresponding MOLOPT basis sets is shown in Figure 1, similar to the procedure we used to optimize lanthanide GTH pseudopotentials and basis sets. ${ }^{41}$ The actinide GTH pseudopotentials were optimized using the ATOM code in the CP2K package (version 6.1). ${ }^{24,43}$ The GTH pseudopotential parameters, within the PBE ${ }^{44}$ formulation, are fitted to reliably describe orbital eigenvalues, charge densities inside $r_{c}$, and wave functions outside $r_{c}$ of the atomic ground states, based on the requirements of norm-conserving pseudopotential. ${ }^{45}$ Despite the great importance of spin-orbital (SO) effects for actinides, our pseudopotential is currently parametrized from scalar relativistic (SR) formalism. The reference data are from scalar relativistic Douglas-Kroll-Hell (DKH) all-electron wave functions. ${ }^{46-49}$ For the fitting process, we used a weighted average penalty function involving Kohn-Sham eigenvalues, radial densities, and location of the radial nodes. Weights, step sizes and convergence values are three main factors affecting optimization of GTH pseudopotentials. We have constructed a general scheme to optimize GTH pseudopotentials by modulating these three factors. A detailed discussion of the optimization process for GTH pseudopotentials is shown in part A of the Supporting Information (SI).

Typical differences in orbital eigenvalues between pseudo-wavefunctions and all-electron KohnSham wavefunctions with $\sim 10^{-5}$ Hartree for valence orbitals, $\sim 10^{-4}$ Hartree for semi-core orbitals and $\sim 10^{-3}$ Hartree for unoccupied orbitals, were met in our optimization as shown in Tables S1 and S2 in part A of the SI. Given the large impact of the $r_{\text {loc }}$ parameter on the fitting procedure with respect to all other parameters, we fitted potentials with different $r_{\mathrm{loc}}$, and then chose the one with the highest degree 
of transferability, i.e. the ability to reproduce a large number of all-electrons results with minimal error. The $r_{\text {loc }}$ values for large-core pseudopotentials vary from $0.45 \AA$ to $0.55 \AA$, and the large-core pseudopotentials with different $r_{\text {loc }}$ for uranium are presented in part F of the SI. Medium-core GTH pseudopotentials were not optimized with different $r_{\text {loc }}$ due to multiple local minima in the fitting process. It is believed that electronic structure calculations are less affected by the hardness of mediumcore pseudopotentials as the short-ranged integral term in local and non-local parts can be analytically calculated with Gaussian basis sets in CP2K software. ${ }^{38}$

Given the fact that basis sets with uncontracted $f$ states improves lanthanide transferability, we investigated the feasibility of uncontracting $f$ states for optimizing MOLOPT basis sets for actinides. ${ }^{41}$ We obtained different Gaussian exponents for $s, p, d$ and $f$ states by using the ATOM code in the CP2K package (version 4.1). To optimize a DZ2P quality basis sets, $[7 s 7 p 7 d 7 f 7 g] /[3 s 2 p 3 d 2 f 1 g]$ contraction for medium-core GTH pseudopotentials and $[7 s 7 p 7 d 7 f 7 g] /[3 s 2 p 2 d 2 f 1 g]$ contraction for large-core GTH pseudopotentials, the contraction coefficients of the $s, p, d, f$ and $g$ states were derived by minimizing a linear combination of energies and condition numbers of the overlap matrix for a set of actinide training molecules. ${ }^{42}$ More details on the MOLOPT basis sets optimization procedure appears in part A of the SI, Tables S3-S6.

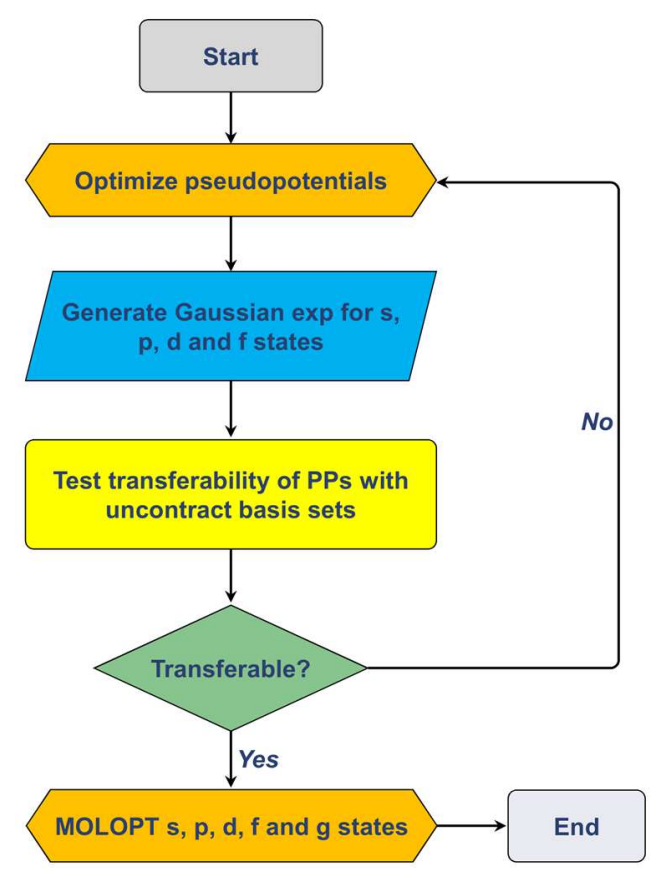

Figure 1. The general procedure to optimize GTH pseudopotentials and MOLOPT basis sets for actinides. 


\subsection{Computational methodology}

The $\mathrm{CP} 2 \mathrm{~K}$ (version 4.1) code was used for benchmarking calculations. ${ }^{24,43}$ The rigorous way to test pseudopotential transferability is by reproducing the reaction energies of redox reactions with changes in actinide oxidation states at the all-electron level. This process allows us to determine if a pseudopotential is able to reproduce the chemistry of distinctly different oxidation states of the pseudoatom such that it can be used in a wide range of chemical environments. Considering the complex character of actinide oxidation states (Figure 2) ${ }^{50}$, and in order to determine pseudopotential transferability, we compared actinide redox reaction energies using the optimized pseudopotentials with those from all-electron calculations using Slater-type basis functions with the quantum chemistry software ADF (version 2016.106). ${ }^{51}$ To cover the most common actinide spins and oxidation states (I, II, ..., VII), we focused on six prototypical reactions:

$$
\begin{gathered}
A n^{I} \mathrm{Cl}+\frac{1}{2} \mathrm{Cl}_{2} \rightarrow A n^{I I} \mathrm{Cl}_{2} \\
A n^{I I} \mathrm{Cl}_{2}+\frac{1}{2} \mathrm{Cl}_{2} \rightarrow A n^{I I I} \mathrm{Cl}_{3} \\
A n^{I I I} \mathrm{Cl}_{3}+\frac{1}{2} \mathrm{Cl}_{2} \rightarrow A n^{I V} \mathrm{Cl}_{4} \\
A n^{I V} \mathrm{Cl}_{4}+\frac{1}{2} \mathrm{Cl}_{2} \rightarrow A n^{V} \mathrm{Cl}_{5} \\
A n^{V} \mathrm{Cl}_{5}+\frac{1}{2} \mathrm{Cl}_{2} \rightarrow A n^{V I} \mathrm{Cl}_{6} \\
A n^{V I} \mathrm{Cl}_{6}+\frac{1}{2} \mathrm{Cl}_{2} \rightarrow A n^{V I I} \mathrm{Cl}_{7}
\end{gathered}
$$




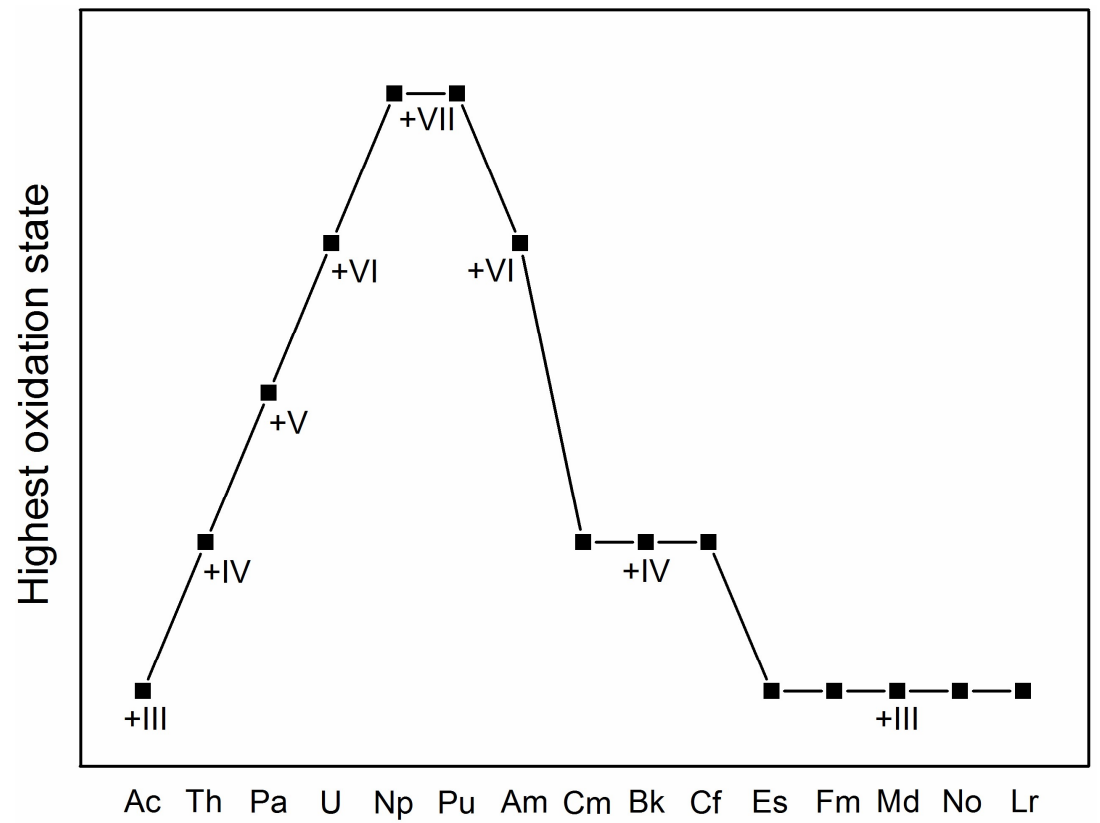

Figure 2. The known highest oxidation states distribution for actinide series.

For each actinide element, we calculated the redox reactions up to the reactions that involve the highest oxidation state of the element as shown in Figure 2, e.g., for uranium the testing redox reactions are $\mathrm{R} 1$ - R5. An ionic model for $\mathrm{AnCl}_{n}(\mathrm{An}=\mathrm{Ac}-\mathrm{Lr}, n=1-7)$ is used where chlorine atoms are considered as closed-shell anions, and the actinide unit includes all the spin density as an actinide cation. ${ }^{41,52}$ The electron configurations of actinide ions from Peterson's work ${ }^{17-18}$ were chosen, and are presented in Table $\mathrm{S} 7$ in part B of the SI. The geometry structures of $\mathrm{AnCl}_{\mathrm{n}}$ and $\mathrm{Cl}_{2}$ were optimized by using Gaussian (version Gaussian09 D.01) at the PBE level. ${ }^{53}$ Scalar relativistic effects were taken into account by using the ECP60MWB effective core potentials (ECP) for actinides. ${ }^{27}$ The ECP60MWB_SEG basis sets for An elements and cc-pVTZ basis sets for $\mathrm{Cl}$ element were used. ${ }^{54-56}$ Vibrational frequency analysis was performed to confirm minima structures on the potential energy surface.

To compare reaction energies consistently, we performed PBE single-point calculations, using $\mathrm{AnCl}_{\mathrm{n}}$ geometries optimized in Gaussian, with ADF using TZ2P quality all-electron Slater-type basis sets for An and $\mathrm{Cl}^{57-58}$ The scalar relativistic effects were included by zero-order regular approximation (ZORA) formalism. ${ }^{59-62}$ A Fermi-Dirac smearing scheme was adopted. The all-electron reference reaction energies of the six redox reactions are depicted in Table S8. To calculate redox energies in $\mathrm{CP} 2 \mathrm{~K}$, molecules were placed in the center of cubic boxes with cell lengths of $20 \AA$ and non-periodic 
condition, which allows us to deal with isolated molecules in a periodic code. A density cutoff of 800 Ry was chosen, based on the testing results with large-core and medium-core pseudopotentials of uranium (Tables S9 - S12 in part B of the SI).

Additional molecular benchmarks to predict molecular structures, bond energies, binding energies, ionization potentials, and enthalpies of formation were also performed with $\mathrm{CP} 2 \mathrm{~K}$ using our optimized pseudopotentials and basis sets. As a reference point, we used either published energy-consistent pseudopotential calculations, or we compared to results from relativistic all-electron reference calculations performed with $\mathrm{ADF}$, as well as to experimental data when available.

\section{RESULTS AND DISCUSSION}

\subsection{Pseudopotential transferability and redox reaction energies}

The 15-element actinide series, from Ac to $\mathrm{Lr}$, has ground states with varying $5 f$ orbital occupation from empty (Ac, Th) to fully filled ( $\mathrm{Lr})$. Generally, the valence electrons occupy $5 f, 6 d$ and $7 s$ orbitals. As shown in Figure 3a, the $7 s$ orbital is the most diffused and fully filled for all actinide ground state atoms. The $6 d$ orbital is occupied with one or two electrons for early actinides (Ac, Th, $\mathrm{Pa}, \mathrm{U}, \mathrm{Np}$ and $\mathrm{Cm}$ ). As is well documented, the actinide $6 s$ and $6 p$ orbitals, extending to valence region (Figure 3a) and significantly participating in chemical bonding, thereby should be treated as semi-core orbitals. ${ }^{63-}$

${ }^{64}$ This effect is even more noticeable for the later actinides owing to $5 f$ orbitals contraction (Figure $3 b$ ).

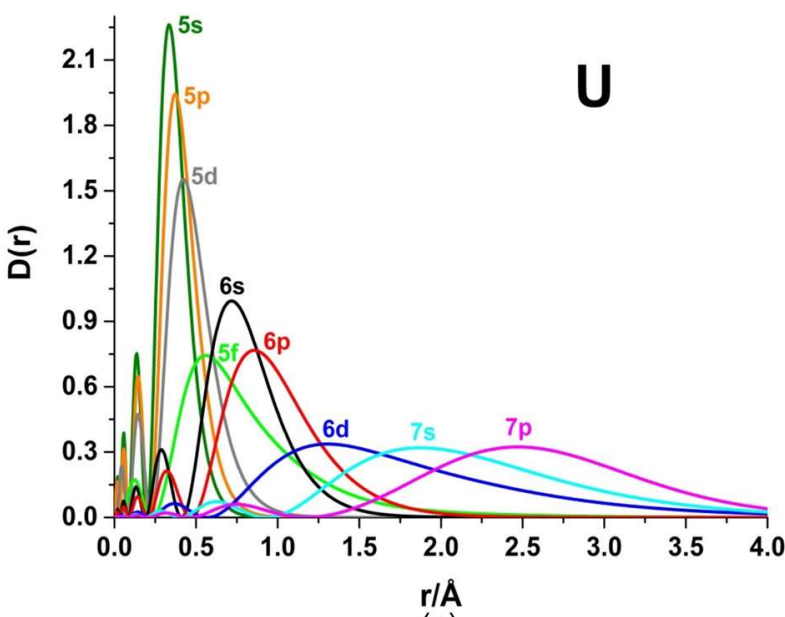

(a)

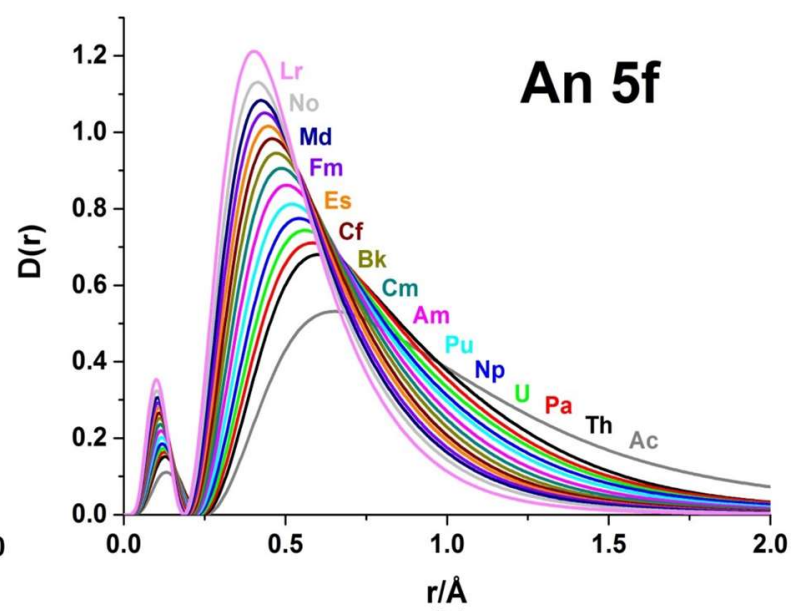

(b)

Figure 3. (a) Radial densities $\mathrm{D}(\mathrm{r})=\mathrm{r}^{2} \mathrm{R}(\mathrm{r})^{2}$ for $5 s, 5 p, 5 d, 6 s, 6 p, 5 f, 6 d$ and $7 s$ orbitals of $\mathrm{U}$ atom. (b) Radial densities $r^{2} R(r)^{2}$ of $5 f$ orbitals from Ac atom to $L r$ atom. $R(r)$ is the radial wavefunction. 
Knowledge of the spatial part and occupation of the orbitals allows us to classify different core type pseudopotentials. For uranium, we define small-core (SC), medium-core (MC) and large-core (LC) pseudopotentials, as shown in Table 2. The concepts of small-core and large-core pseudopotentials for actinides were mainly proposed by Dolg and co-workers. ${ }^{26-30}$ The large-core pseudopotential scheme is straightforward, as only $6 s$ and $6 p$ orbitals are included in the semi-core region. In the small-core pseudopotentials, the orbitals with same main quantum number $(5 s, 5 p$ and $5 d)$ are treated as semi-core orbitals due to the spatial overlap between $5 f$ and $5 d$ orbitals (Figure 3a). Krack and Smirnov have published two large-core GTH pseudopotentials for uranium. ${ }^{33-34}$ As a starting point, we tested their transferability of large-core pseudopotentials and we found that they can replicate redox reaction energies for the lower (I - III) oxidation states only, see Table 3 and discussion below. Clearly pseudopotentials for higher oxidation states are still needed.

Table 2. The electronic configurations of core, semi-core and valence regions for small-core, mediumcore and large-core pseudopotentials for uranium.

\begin{tabular}{cccc}
\hline \hline Core type & Core $^{\mathrm{a}}$ & Semi-core & Valence \\
\hline Small-core & {$[\mathrm{Kr}] 4 \mathrm{~d}^{10} 4 \mathrm{f}^{14}$} & $5 \mathrm{~s}^{2} 5 \mathrm{p}^{6} 5 \mathrm{~d}^{10} 6 \mathrm{~s}^{2} 6 \mathrm{p}^{6}$ & $5 \mathrm{f}^{3} 6 \mathrm{~d}^{1} 7 \mathrm{~s}^{2}$ \\
Medium-core & {$[\mathrm{Xe}] 4 \mathrm{f}^{14}$} & $5 \mathrm{~d}^{10} 6 \mathrm{~s}^{2} 6 \mathrm{p}^{6}$ & $5 \mathrm{f}^{3} 6 \mathrm{~d}^{1} 6 \mathrm{~s}^{2}$ \\
Large-core & {$[\mathrm{Xe}] 4 \mathrm{f}^{14} 5 \mathrm{~d}^{10}$} & $6 \mathrm{~s}^{2} 6 \mathrm{p}^{6}$ & $5 \mathrm{f}^{3} 6 \mathrm{~d}^{1} 6 \mathrm{~s}^{2}$ \\
\hline
\end{tabular}

a $[\mathrm{Kr}]$ and $[\mathrm{Xe}]$ mean the electronic configurations of $\mathrm{Ar}$ and $\mathrm{Kr}$ atoms.

Small-core pseudopotentials usually produce more accurate results, and need larger computational cost. $^{26-27,}$ 29-30 Excellent agreement with all-electron results is achieved when using small-core pseudopotentials, as shown in Table 3. The absolute errors for R1-R6 reactions are $<2.0 \mathrm{kcal} / \mathrm{mol}$, confirming that the $5 s, 5 p$ and $5 d$ orbitals should be in the semi-core region. However, pseudopotentials with too many semi-core wave functions are computationally more expensive. A plausible compromise is to include $5 d^{10}$ into the semi-core region to form medium-core GTH pseudopotentials (Table 2), based on the existing large-core GTH pseudopotentials. Including the $5 d$ electrons in the semi-core is a practical way to balance chemical accuracy and computational cost, but it requires an increased number of planewaves and/or a finer mesh in the real space grid to properly expand the density. Our newly optimized medium-core GTH pseudopotentials (abbreviated as AnPP-M) and large-core GTH 
pseudopotentials (abbreviated as AnPP-L), and accompanying MOLOPT basis sets (abbreviated as AnBS-M and AnBS-L) are reported in part F of the SI.

Table 3. Reaction energies (kcal/mol) with previously existing large-core GTH pseudopotentials and energy-consistent pseudopotentials for uranium at the PBE level. Errors with respect to the all-electron reaction energies calculated with $\mathrm{ADF}$ are in parenthesis.

\begin{tabular}{cccccc}
\hline \hline PPs & $\mathrm{R}^{\mathrm{d}}$ & $\mathrm{R} 2^{\mathrm{d}}$ & $\mathrm{R}^{\mathrm{d}}$ & $\mathrm{R} 4^{\mathrm{d}}$ & $\mathrm{R}^{\mathrm{d}}$ \\
\hline Krack_PP $^{\mathrm{a}}$ & $-98.8(-12.8)$ & $-86.9(-4.5)$ & $-54.8(15.7)$ & $-23.7(19.1)$ & $-12.9(22.0)$ \\
Smirnov_PP $^{\mathrm{b}}$ & $-117.5(-31.6)$ & $-100.5(-18.0)$ & $-59.0(11.5)$ & $-20.5(22.3)$ & $-8.0(26.9)$ \\
ECP78MWB $^{\mathrm{c}}$ & $-93.7(-7.8)$ & $-93.3(-10.9)$ & $-99.1(-28.6)$ & $-62.4(-19.6)$ & $-65.5(-30.6)$ \\
ECP60MWB $^{\mathrm{c}}$ & $-84.6(1.3)$ & $-83.3(-0.8)$ & $-69.0(1.6)$ & $-41.5(1.3)$ & $-33.2(1.7)$ \\
\hline
\end{tabular}

${ }^{\text {a }}$ Krack_PP is large-core GTH pseudopotentials of uranium optimized by Krack.

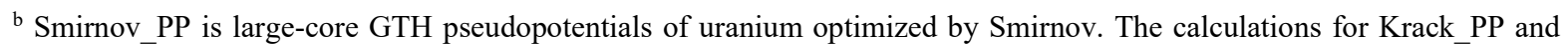
Smornov_PP were performed by using CP2K.

${ }^{\mathrm{c}}$ ECP78MWB (large-core) and ECP60MWB (small-core) are energy-consistent pseudopotentials optimized by Dolg and coworkers. The calculations for these two pseudopotentials were performed by using Gaussian.

${ }^{\mathrm{d}}$ Error $=\left(\mathrm{E}_{\mathrm{CP} 2 \mathrm{~K}}-\mathrm{E}_{\mathrm{ADF}}\right)$. Reaction energy is outside parenthesis, and error is inside parenthesis hereafter.

Due to the "plutonium turn" phenomenon in the actinide series, ${ }^{65}$ we split the actinide series into two sets to facilitate discussion of transferability results, since the early actinides (Ac $-\mathrm{Cm}$ ) can achieve high oxidation states (Figure 2), while the later ones (Bk - Lr) prefer the lower oxidation states. The redox reaction energies computed with our optimized AnPP-L pseudopotentials, are presented in Tables $4(\mathrm{Ac}-\mathrm{Cm})$ and $5(\mathrm{Bk}-\mathrm{Lr})$. We note that the large-core pseudopotentials do not perform as well for the later elements in the series and high oxidation states for the early actinides. 
Table 4. Reaction energies (kcal/mol) computed with our optimized AnPP-L pseudopotentials for Ac to $\mathrm{Cm}$ at the PBE level. Errors with respect to all-electron reaction energies are in parenthesis.

\begin{tabular}{ccccccc}
\hline \hline Element & $\mathrm{R} 1$ & $\mathrm{R} 2$ & $\mathrm{R} 3$ & $\mathrm{R} 4$ & $\mathrm{R} 5$ & $\mathrm{R} 6$ \\
\hline $\mathrm{Ac}$ & $-80.2(-0.2)$ & $-83.7(0.0)$ & & & & \\
$\mathrm{Th}$ & $-80.7(10.0)$ & $-94.8(2.9)$ & $-91.9(-1.2)$ & & & \\
$\mathrm{Pa}$ & $-84.5(3.5)$ & $-84.7(4.0)$ & $-79.9(8.5)$ & $-38.3(17.7)$ & & \\
$\mathrm{U}$ & $-85.4(0.6)$ & $-81.8(0.6)$ & $-60.4(10.1)$ & $-28.5(14.3)$ & $-16.3(18.6)$ & \\
$\mathrm{Np}$ & $-87.1(-0.8)$ & $-79.6(-3.2)$ & $-42.6(12.7)$ & $-5.0(10.6)$ & $-26.5(13.2)$ & $55.1(18.2)$ \\
$\mathrm{Pu}$ & $-72.1(2.7)$ & $-65.7(-1.1)$ & $-38.0(4.0)$ & $-3.3(10.1)$ & $-3.7(9.3)$ & $43.0(15.6)$ \\
$\mathrm{Am}$ & $-69.4(0.1)$ & $-47.7(-1.3)$ & $-17.9(2.8)$ & $-1.8(3.9)$ & $0.6(5.5)$ & \\
$\mathrm{Cm}$ & $-66.6(1.6)$ & $-92.3(-10.0)$ & $-6.8(-0.1)$ & & & 16.9 \\
\hline $\mathrm{MAD}^{\mathrm{a}}$ & 2.4 & 2.9 & 5.6 & 11.3 & 11.6 & \\
\hline
\end{tabular}

${ }^{a}$ Mean absolute deviation hereafter. $\mathrm{MAD}=\sum_{i}\left|E_{i}\right| / \mathrm{N}$ where $\mathrm{E}_{\mathrm{i}}$ is the error for each actinide element, and $\mathrm{N}$ is the number of actinide elements.

Table 5. Reaction energies (kcal/mol) computed with our optimized AnPP-L pseudopotentials for Bk to $\mathrm{Lr}$ at the PBE level. Errors with respect to all-electron reaction energies are in parenthesis.

\begin{tabular}{cccc}
\hline \hline Element & R1 & R2 & R3 \\
\hline Bk & $-63.2(5.6)$ & $-82.5(-16.2)$ & $-51.6(-11.5)$ \\
Cf & $-68.4(-0.3)$ & $-70.1(-19.3)$ & $-32.1(-8.7)$ \\
Es & $-70.0(-2.7)$ & $-53.9(-12.7)$ & \\
Fm & $-72.1(-5.5)$ & $-53.0(-21.2)$ & \\
Md & $-70.1(-3.5)$ & $-26.7(-8.5)$ & \\
No & $-66.6(-2.4)$ & $-18.0(-9.8)$ & \\
Lr & $-58.8(-3.3)$ & $-77.6(-3.0)$ & \\
\hline MAD & 3.4 & 13.0 & 10.1 \\
\hline
\end{tabular}

The AnPP-L pseudopotentials can reproduce redox reaction energies with respect to all-electron calculations for the lower oxidation states. As shown in Tables 4 and 5, mean absolute deviation (MAD) values $<6 \mathrm{kcal} / \mathrm{mol}$ are only observed for reactions R1 - R3 for the early actinides, and R1 for the later 
elements. The reaction energies of high oxidation states redox reactions (R4 - R6) for early actinides are systematically underestimated with comparison to ADF results as shown in Figure S5. This implies that, to accurately describe the high oxidation states of actinides, the inclusion of deeper lying states is necessary, invoking the use of medium-core or even small-core pseudopotentials.

The redox reaction energies using our medium-core AnPP-M pseudopotentials are listed in Tables 6 and 7. The reaction energies of AnPP-M pseudopotentials for uranium in Table 6 are in good agreement with small-core energy-consistent pseudopotentials results (Table 3), thus demonstrating that both small-core and medium-core pseudopotentials can be highly transferable. ${ }^{29}$ The error with respect to all-electron calculations of the R1-R6 reaction energies calculated with the AnPP-M pseudopotentials are acceptable $(<5 \mathrm{kcal} / \mathrm{mol})$, and considerably better than those calculated with the AnPP-L pseudopotentials (Figure 4). Notably, the R3 - R6 reaction energies are significantly improved for the early actinides with MAD reduced to only $\sim 2 \mathrm{kcal} / \mathrm{mol}$.

Table 6. Reaction energies ( $\mathrm{kcal} / \mathrm{mol}$ ) computed with our optimized AnPP-M pseudopotentials for Ac to $\mathrm{Cm}$ at the PBE level. Errors with respect to all-electron reaction energies are in parenthesis.

\begin{tabular}{ccccccc}
\hline \hline Element & R1 & R2 & R3 & R4 & R5 & R6 \\
\hline Ac & $-78.6(1.3)$ & $-84.7(-1.0)$ & & & & \\
$\mathrm{Th}$ & $-93.9(-3.3)$ & $-100.8(-3.1)$ & $-93.3(-2.6)$ & & & \\
$\mathrm{Pa}$ & $-90.5(-2.5)$ & $-91.3(-2.6)$ & $-88.1(0.4)$ & $-56.6(-0.7)$ & & \\
$\mathrm{U}$ & $-86.9(-1.0)$ & $-85.0(-2.5)$ & $-70.2(0.3)$ & $-42.3(0.5)$ & $-35.3(-0.4)$ & \\
$\mathrm{Np}$ & $-90.7(-4.4)$ & $-81.8(-5.4)$ & $-55.3(0.1)$ & $-19.1(-3.6)$ & $-37.2(2.5)$ & $36.2(-0.7)$ \\
$\mathrm{Pu}$ & $-79.1(-4.2)$ & $-69.7(-5.0)$ & $-46.0(-4.1)$ & $-15.3(-1.9)$ & $-16.6(-3.6)$ & $26.8(-0.6)$ \\
$\mathrm{Am}$ & $-73.1(-3.6)$ & $-51.1(-4.7)$ & $-21.3(-0.6)$ & $-9.7(-4.1)$ & $-7.2(-2.3)$ & \\
$\mathrm{Cm}$ & $-69.5(-1.3)$ & $-87.3(-5.1)$ & $-8.5(-1.8)$ & & & 0.7 \\
\hline $\mathrm{MAD}$ & 2.7 & 3.7 & 1.4 & 2.1 & 2.2 & \\
\hline
\end{tabular}


Table 7. Reaction energies ( $\mathrm{kcal} / \mathrm{mol})$ computed with our optimized AnPP-M pseudopotentials for Bk to $\mathrm{Lr}$ at the PBE level. Errors with respect to all-electron reaction energies are in parenthesis.

\begin{tabular}{cccc}
\hline \hline Element & R1 & R2 & R3 \\
\hline Bk & $-69.5(-0.7)$ & $-73.6(-7.3)$ & $-43.4(-3.2)$ \\
Cf & $-70.3(-2.3)$ & $-57.9(-7.0)$ & $-27.5(-4.0)$ \\
Es & $-72.8(-5.5)$ & $-44.8(-3.6)$ & \\
Fm & $-72.7(-6.2)$ & $-38.0(-6.1)$ & \\
Md & $-72.0(-5.5)$ & $-22.6(-4.4)$ & \\
No & $-66.6(-2.4)$ & $-13.7(-5.5)$ & \\
Lr & $-52.3(3.2)$ & $-74.2(0.3)$ & \\
\hline MAD & 3.2 & 4.9 & 3.6 \\
\hline
\end{tabular}

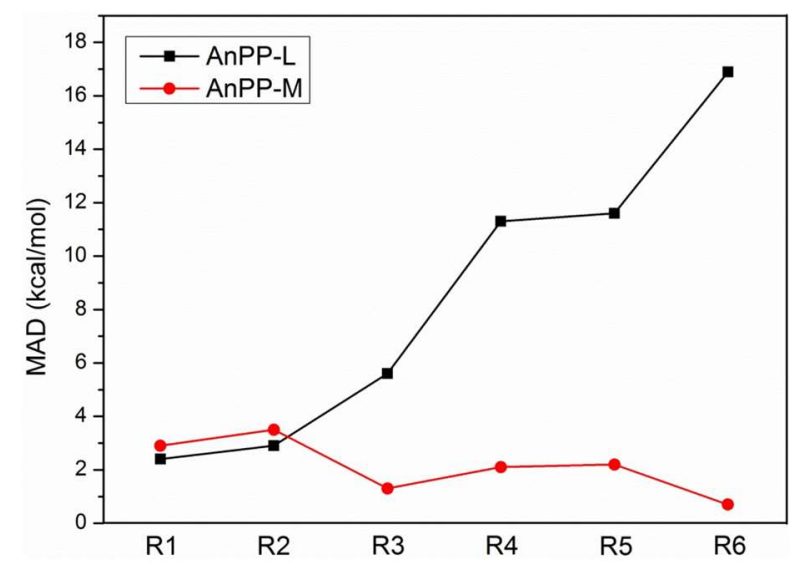

(a)

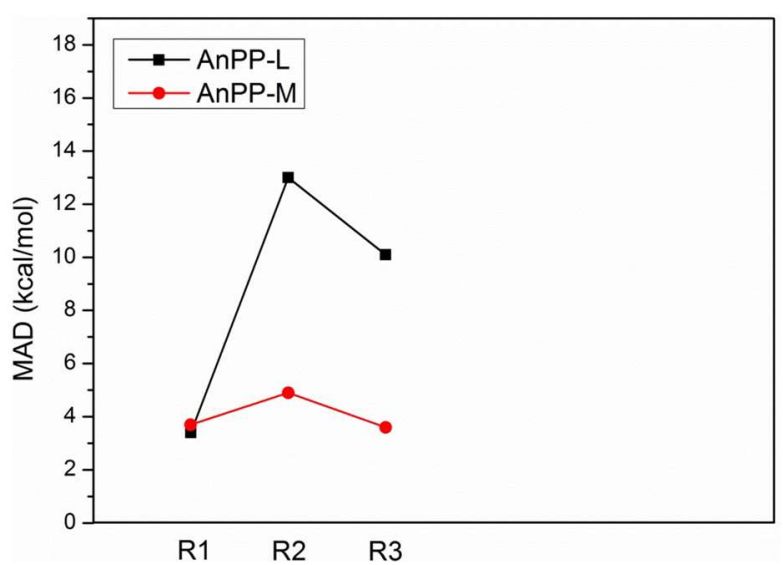

(b)

Figure 4. The mean absolute deviation (MAD) values of reaction energies of redox reactions using CP2K with our AnPP-L and AnPP-M pseudopotentials and basis sets with respect to all-electron ADF calculations at the PBE level. For (a), the actinide elements are from Ac to Cm; for (b), the actinide elements are from Bk to Lr.

We compared the redox reaction energies computed with our norm-conserving pseudopotentials with those calculated with energy-consistent pseudopotentials, that are widely used by the molecular quantum chemistry community. The reaction energies of large-core and small-core energy-consistent pseudopotentials are presented in Tables S17 - S19. The large-core energy-consistent pseudopotentials overestimate all-electron reaction energy results compared to the results using the AnPP-L 
pseudopotentials (Table S17). For early actinides (Ac - Cm), small-core energy-consistent pseudopotentials yield R1 - R6 reaction energies that are closer to all-electron results than our AnPPM pseudopotentials (Table S18), especially for the R1 and R2 reactions. Although optimizing smallcore norm-conserving GTH pseudopotentials would improve transferability results, these would become too computationally expensive for the simulation of large-scale actinide-containing systems, especially when involving condensed phase.

A key question remains to be answered: Why are medium-core GTH pseudopotentials needed to properly describe the high oxidation states for the early actinides and nearly all oxidation states for the late actinides? To answer this question, we plot in Figure 5 the radial densities for the $5 s, 5 p, 5 d$ and $5 f$ orbitals of uranium in different oxidation states. It turns out that with increasing of oxidation states of uranium, while the $R_{\max }$ of the $5 s, 5 p$, and $5 d$ orbitals barely change, the $5 f$ orbital becomes more compact for higher oxidation states of uranium, as previously discussed by Dolg et al. ${ }^{29}$ and us. ${ }^{66}$ The $R_{\max }$ of the uranium $5 f$ orbital is nearly the same $(\sim 0.56 \AA)$ in the 0 , I and II oxidation states, however, they shrink in $\sim 0.01 \AA$ increments starting at the III oxidation state. At the highest oxidation states, the radial maximum of the $5 f$ orbital approaches that of the $5 d$ orbital, indicating that these states interact/repel each other and hence a polarization of the $5 f$ orbital become more problematic for the higher oxidation states. This effect likely explains the observation that early actinides require mediumcore pseudopotentials for high oxidation states redox reactions. 


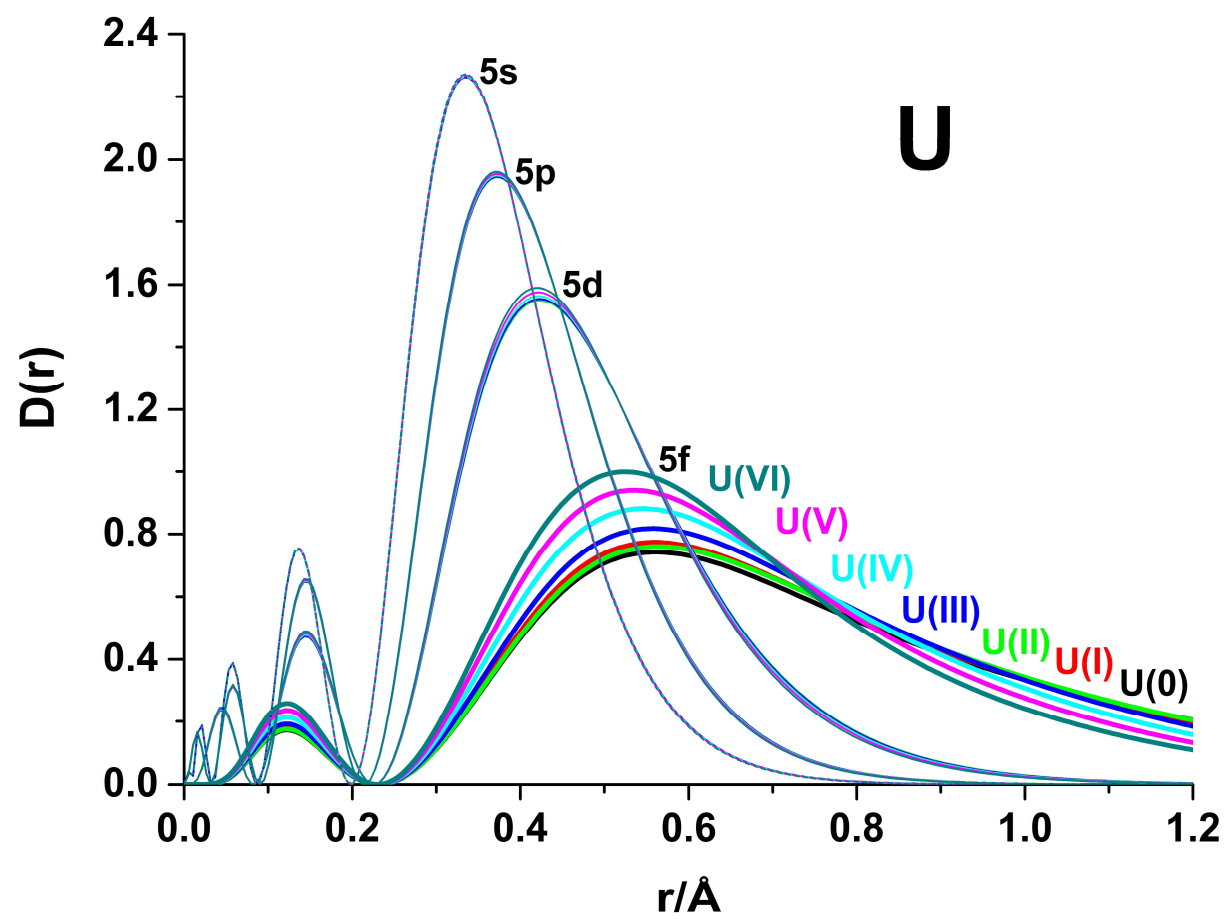

Figure 5. Radial densities $\mathrm{D}(\mathrm{r})=\mathrm{r}^{2} \mathrm{R}(\mathrm{r})^{2}$ for $5 s, 5 p, 5 d$, and $5 f$ orbitals of atomic ions $\mathrm{U}^{+}-\mathrm{U}^{6+}$.

Pseudopotential transferability of the later actinides is also affected by the spatial correlation between $5 f$ orbital and $5 d$ orbital. To understand this effect, we plot the radii of maximum radial densities $\left(R_{\max }\right)$ of $5 s, 5 p, 5 d$ and $5 f$ orbitals of actinide atom (Figure 6) derived from numerical relativistic Dirac-Fock calculations.$^{67}$ There is a significant separation between the radial maxima of $5 d$, $5 f$ and $5 s, 5 p$ orbitals, demonstrating the validity of medium-core pseudopotentials for the actinide series. As the series progresses, the $5 f$ orbitals become spatially closer to $5 d$ orbitals such that by the end of the series $R_{\max }$ for the two orbitals is only about $0.06 \AA$ apart, indicating increased interaction between the two. The spatial correlation between $5 f$ and $5 d$ orbitals in the later actinides, although not as marked as the overlap between $4 f$ and $4 d$ orbitals for the later lanthanides, is a likely reason why medium-core GTH pseudopotentials are also required for the late actinides. ${ }^{41}$ 


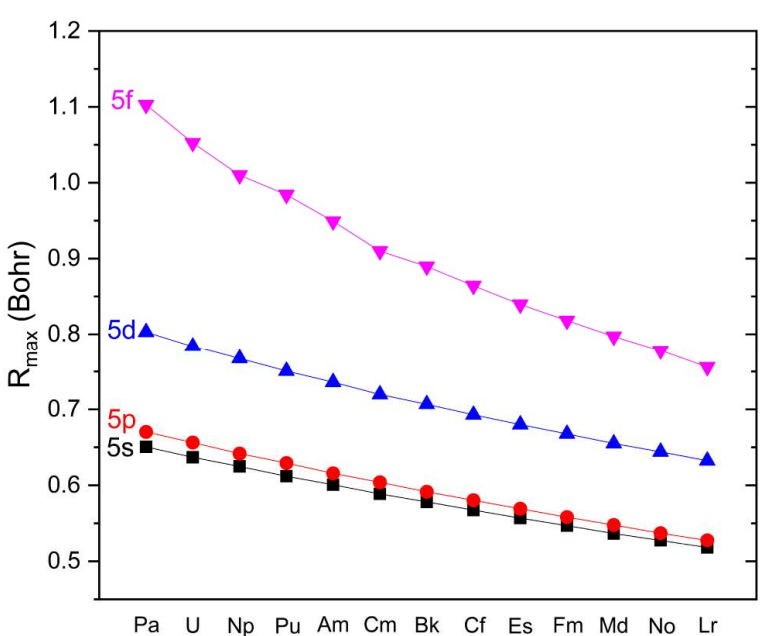

(a)

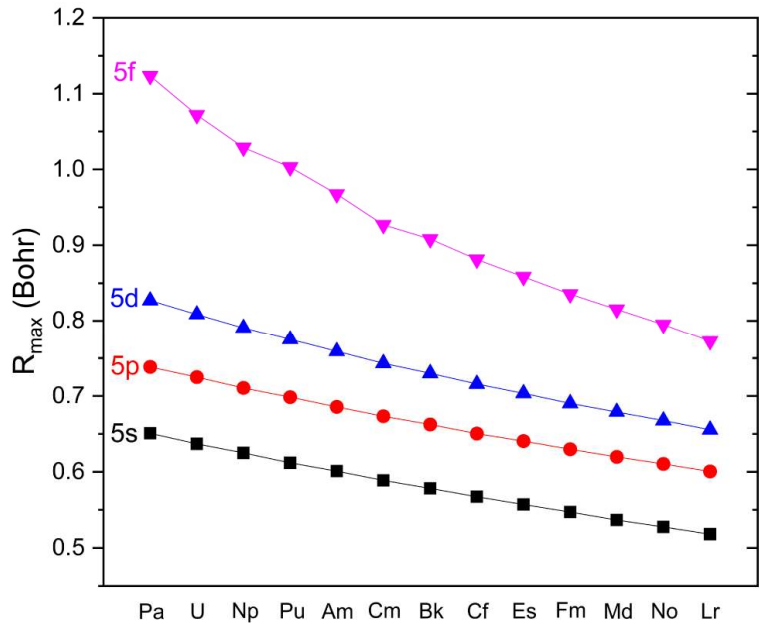

(b)

Figure 6. The $\mathrm{R}_{\max }$ of $5 s, 5 p, 5 d$ and $5 f$ orbitals for actinide series derived from numerical Dirac-Fock calculations. ${ }^{65}$ For $(\mathrm{a}), j=l-\frac{1}{2}$; for $(\mathrm{b}), j=l+\frac{1}{2}$.

Overall, the transferability of pseudopotentials for heavy elements is dictated by oxidation states and spatial correlation of $d$ and $f$ orbitals. Since lanthanides overwhelmingly favor the III oxidation state with some exceptions, ${ }^{68-70}$ their pseudopotential transferability is mainly affected by the spatial correlation of the $4 d$ and $4 f$ orbitals for late lanthanides. Hence, in our previous work we successfully adopted large-core pseudopotentials for early lanthanides ( $\mathrm{La}-\mathrm{Gd})$ and medium-core pseudopotentials for late lanthanides $(\mathrm{Tb}-\mathrm{Lu}) .{ }^{41}$ For actinides, since the early elements favor a variety of oxidation states, medium-core pseudopotentials are required to treat reactions involving high oxidation states. The late actinides, akin to the late lanthanides, also require medium-core pseudopotentials due to spatial overlap between the $5 d$ and $5 f$ orbitals.

As a related test of the transferability principle, we consider the direct computation of ionization potentials for An atoms and ions. The first two ionization potentials (IPs) of actinides were, computed as the difference between the ground state energies of $\mathrm{An}^{\mathrm{q}^{+}}$and $\mathrm{An}^{(\mathrm{q}+1)+}$, where $\mathrm{q}$ denotes charge. Although our procedure is not based on state energies, it is a simple and fast procedure to calculate ionization potential by using density functional theory at the PBE level. The computed first and second ionization potential of actinide atoms using our AnPP-L and AnPP-M pseudopotentials and basis sets are presented in Tables 8 and 9. Consistent with the above analysis, both AnPP-L and AnPP-M can reproduce first and second ionization potentials, with MAD values $<10 \mathrm{kcal} / \mathrm{mol}$ compared to experiment and a maximum error of $24 \mathrm{kcal} / \mathrm{mol}$. 
Table 8. Composite results for the first ionization potentials of actinide atoms (unit in $\mathrm{kcal} / \mathrm{mol}$ ).

\begin{tabular}{cccccc}
\hline \hline Element & AnPP-L & AnPP-M & Exp. $^{\mathrm{a}}$ & Error(L) & Error(M) $^{\mathrm{b}}$ \\
\hline $\mathrm{Ac}$ & 130.1 & 121.7 & 124.1 & $6.0(5 \%)$ & $-2.4(2 \%)$ \\
$\mathrm{Th}$ & 133.7 & 136.6 & 145.4 & $-11.7(8 \%)$ & $-8.8(6 \%)$ \\
$\mathrm{Pa}$ & 138.9 & 129.4 & 135.8 & $3.1(2 \%)$ & $-6.4(5 \%)$ \\
$\mathrm{U}$ & 140.4 & 131.0 & 142.8 & $-2.4(2 \%)$ & $-11.8(8 \%)$ \\
$\mathrm{Np}$ & 131.3 & 121.0 & 144.5 & $-13.2(9 \%)$ & $-23.5(16 \%)$ \\
$\mathrm{Pu}$ & 129.3 & 125.4 & 139.0 & $-9.7(7 \%)$ & $-13.6(10 \%)$ \\
$\mathrm{Am}$ & 127.2 & 126.1 & 137.8 & $-10.6(8 \%)$ & $-11.7(8 \%)$ \\
$\mathrm{Cm}$ & 133.8 & 114.3 & 138.2 & $-4.4(3 \%)$ & $-23.9(17 \%)$ \\
$\mathrm{Bk}$ & 136.0 & 140.4 & 142.9 & $-6.9(5 \%)$ & $-2.5(2 \%)$ \\
$\mathrm{Cf}$ & 138.9 & 140.6 & 144.9 & $-6.0(4 \%)$ & $-4.3(3 \%)$ \\
$\mathrm{Es}$ & 140.1 & 138.4 & 146.8 & $-6.7(5 \%)$ & $-8.4(6 \%)$ \\
$\mathrm{Fm}$ & 144.0 & 141.7 & 149.9 & $-5.9(4 \%)$ & $-8.2(5 \%)$ \\
$\mathrm{Md}$ & 146.6 & 145.4 & 151.7 & $-5.1(3 \%)$ & $-6.3(4 \%)$ \\
$\mathrm{No}$ & 149.4 & 152.3 & 153.4 & $-4.0(3 \%)$ & $-1.1(1 \%)$ \\
$\mathrm{Lr}$ & 101.8 & 100.1 & 114.4 & $-12.6(11 \%)$ & $-14.3(13 \%)$ \\
\hline & & $\mathrm{MAD}$ & & $7.2(5 \%)$ & $9.8(7 \%)$ \\
\hline
\end{tabular}

${ }^{a}$ Experimental values are taken from the NIST database. ${ }^{71}$

${ }^{\mathrm{b}}$ Absolute error Error(L) is calculated as Error(L) $=\mathrm{IP} 1_{\mathrm{AnPP}-\mathrm{L}}-\mathrm{IP} 1_{\text {Exp. }}$ and Error(M) is calculated as Error $(\mathrm{M})=$ IP1 $1_{\text {AnPP-M }}$ - IP1 $1_{\text {Exp. }}$ Relative error is in parenthesis. 
Table 9. Composite results for the second ionization potentials of actinide atoms (unit in $\mathrm{kcal} / \mathrm{mol}$ ).

\begin{tabular}{cccccc}
\hline \hline Element & AnPP-L & AnPP-M & Exp. $^{\mathrm{a}}$ & Error(L) & Error(M) \\
\hline $\mathrm{Ac}$ & 270.9 & 273.0 & 271.1 & $-0.2(0 \%)$ & $1.9(1 \%)$ \\
$\mathrm{Th}$ & 271.7 & 276.7 & 279.0 & $-7.3(3 \%)$ & $-2.3(1 \%)$ \\
$\mathrm{Pa}$ & 262.9 & 271.6 & 268.0 & $-5.1(2 \%)$ & $3.6(1 \%)$ \\
$\mathrm{U}$ & 256.6 & 267.0 & 268.0 & $-11.4(4 \%)$ & $-1.0(0 \%)$ \\
$\mathrm{Np}$ & 273.4 & 274.4 & 267.0 & $6.4(2 \%)$ & $7.4(3 \%)$ \\
$\mathrm{Pu}$ & 275.6 & 278.1 & 265.0 & $10.6(4 \%)$ & $13.1(5 \%)$ \\
$\mathrm{Am}$ & 280.1 & 281.1 & 269.0 & $11.1(4 \%)$ & $12.1(4 \%)$ \\
$\mathrm{Cm}$ & 289.8 & 288.4 & 286.0 & $3.8(1 \%)$ & $2.4(1 \%)$ \\
$\mathrm{Bk}$ & 286.8 & 284.7 & 276.0 & $10.8(4 \%)$ & $8.7(3 \%)$ \\
$\mathrm{Cf}$ & 288.2 & 288.1 & 278.0 & $10.2(4 \%)$ & $10.1(4 \%)$ \\
$\mathrm{Es}$ & 290.0 & 291.3 & 281.0 & $9.0(3 \%)$ & $10.3(4 \%)$ \\
$\mathrm{Fm}$ & 292.9 & 294.1 & 285.0 & $7.9(3 \%)$ & $9.1(3 \%)$ \\
$\mathrm{Md}$ & 294.4 & 296.2 & 287.0 & $7.4(3 \%)$ & $9.2(3 \%)$ \\
$\mathrm{No}$ & 297.0 & 296.1 & 290.0 & $7.0(2 \%)$ & $6.1(2 \%)$ \\
$\mathrm{Lr}$ & 336.4 & 338.8 & 328.0 & $8.4(3 \%)$ & $10.8(3 \%)$ \\
\hline & & $\mathrm{MAD}$ & & $7.8(3 \%)$ & $7.2(3 \%)$ \\
\hline
\end{tabular}

${ }^{\text {a }}$ Experimental values are taken from the NIST database. ${ }^{71}$

${ }^{\mathrm{b}}$ Absolute error Error(L) is calculated as Error(L) $=$ IP1 $1_{\mathrm{AnPP}-\mathrm{L}}-\mathrm{IP} 1_{\text {Exp. }}$ and Error(M) is calculated as Error(M) $=$ IP1 $1_{\text {AnPP-M }}$ - IP1 $1_{\text {Exp. }}$ Relative error is in parenthesis.

\subsection{Molecular benchmarks}

Besides the testing of the atomic properties, a variety of molecular benchmark calculations are performed to further validate the accuracy of our actinide GTH pseudopotentials and MOLOPT basis sets.

\subsubsection{Molecular structures}

As a first evaluation, the molecular structures (bond lengths and angles) of actinide halides with 
III, V and VI oxidations states for actinides and actinide monohydrates with III oxidation state for actinides (Figure 7), are optimized. The $\mathrm{AnX}_{5}$ molecule has two possible structures, with $\mathrm{C}_{4 \mathrm{v}}$ and $\mathrm{D}_{3 \mathrm{~h}}$ symmetries. Although ab initio studies with a high level theory showed that the energy of the $\mathrm{UCl}_{5} \mathrm{D}_{3 \mathrm{~h}}$ structure is energetically favored by $1 \mathrm{kcal} / \mathrm{mol},{ }^{17,}{ }^{28}$ we chose the $\mathrm{C}_{4 \mathrm{v}}$ structure for $\mathrm{AnX}_{5}$ in our calculations due to the detection of $\mathrm{C}_{4 \mathrm{v}}$ symmetry for $\mathrm{UCl}_{5}$ through infrared spectrum. ${ }^{72}$ Noting that there are limited experimental results for structure parameters for the whole actinide series, we compare our results with all-electron calculations.

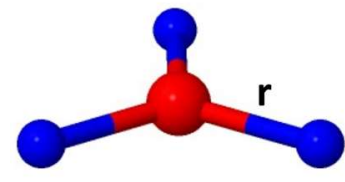

$$
\mathrm{AnX}_{3}(\mathrm{An}=\mathrm{Ac}-\mathrm{Lr} \text {, }
$$
$\mathrm{X}=\mathrm{F}, \mathrm{Cl})$

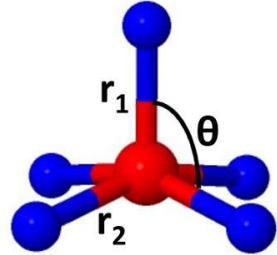

$$
\begin{gathered}
A n X_{5}(A n=P a-A m, \\
X=F, C l)
\end{gathered}
$$

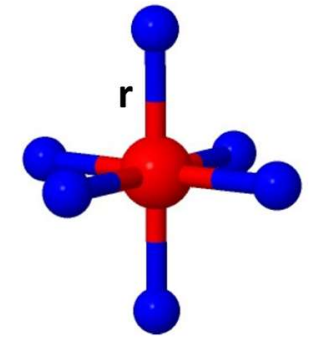

$A n X_{6}(A n=U-A m$, $\mathrm{X}=\mathrm{F}, \mathrm{Cl})$

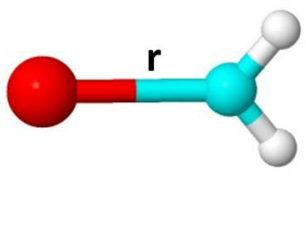

$\operatorname{An}\left(\mathrm{H}_{2} \mathrm{O}\right)^{3+}$

$(\mathrm{An}=\mathrm{Ac}-\mathrm{Cf})$

Figure 7. Molecular structures and geometry parameters of actinide halides with III, V and VI oxidations states for actinides and actinide monohydrates with III oxidation state for actinides

Specific bond lengths and angles of the actinide molecules shown in Figure 7, calculated with the AnPP-M and AnPP-L pseudopotentials, as well as all-electron calculation, are presented in Tables S20 - S28. Mean average deviations (MAD) values across the series for the AnPP-L and AnPP-M calculation with respect to all-electron calculations appear in Table 10. Not surprisingly, the AnPP-M results are in better agreement with all-electron reference data, as also shown in Figure 8.

The actinide contraction $\Delta_{\mathrm{An}}$, quantified by the An-F bond length difference between $\mathrm{AcF}_{3}$ and $\mathrm{LrF}_{3}$, are $0.152 \AA$ and $0.160 \AA$ calculated with AnPP-L and AnPP-M pseudopotentials, respectively. These results are in good agreement with previous theoretical results using energy-consistent pseudopotentials $(0.17 \AA$ and $0.18 \AA){ }^{73}$ The actinide contractions of $0.206 \AA$ and $0.190 \AA$ in $\mathrm{AnCl}_{3}$, and of $0.194 \AA$ and $0.203 \AA$ in $\mathrm{An}\left(\mathrm{H}_{2} \mathrm{O}\right)^{3+}$, with the AnPP-L and AnPP-M pseudopotentials respectively, are considerably larger in comparison to contractions calculated with $\mathrm{AnF}_{3}$. An-F bonds are more rigid than the $\mathrm{An}-\mathrm{Cl}$ and $\mathrm{An}-\mathrm{O}$ bonding interactions, and as such inducing the difference on actinide contraction. ${ }^{73-75}$ For actinide molecules with higher V and VI oxidation states for actinides, the bond 
length errors calculated using the AnPP-M pseudopotentials are smaller than those calculated with the AnPP-L pseudopotentials, which agrees with our results on the transferability of medium-core pseudopotentials for actinides.

Table 10. Computed MAD values for bond length $(\AA)$ of $A n-X(X=F, C l, O)$ in actinide halides with III, V and VI oxidation states for actinides and actinide monohydrate with III oxidation state for actinides using $\mathrm{CP} 2 \mathrm{~K}$ with our AnPP-L and AnPP-M pseudopotentials and basis sets with respect to all-electron ADF calculations at the PBE level.

\begin{tabular}{ccc}
\hline & \multicolumn{2}{c}{$\mathrm{MAD}(\AA)$} \\
\cline { 2 - 3 } & $\Delta \mathrm{L}_{\text {AnPP-L - AE }}$ & $\Delta \mathrm{L}_{\text {AnPP-M - AE }}$ \\
\hline $\mathrm{AnF}_{3}$ & 0.042 & 0.012 \\
$\mathrm{AnF}_{5}$ & 0.021 & 0.008 \\
$\mathrm{AnF}_{6}$ & 0.024 & 0.003 \\
$\mathrm{An} \mathrm{H}_{2} \mathrm{O}^{3+}$ & 0.039 & 0.026 \\
$\mathrm{AnCl}_{3}$ & 0.025 & 0.007 \\
$\mathrm{AnCl}_{5}$ & 0.031 & 0.003 \\
$\mathrm{AnCl}_{6}$ & 0.041 & 0.003 \\
\hline
\end{tabular}

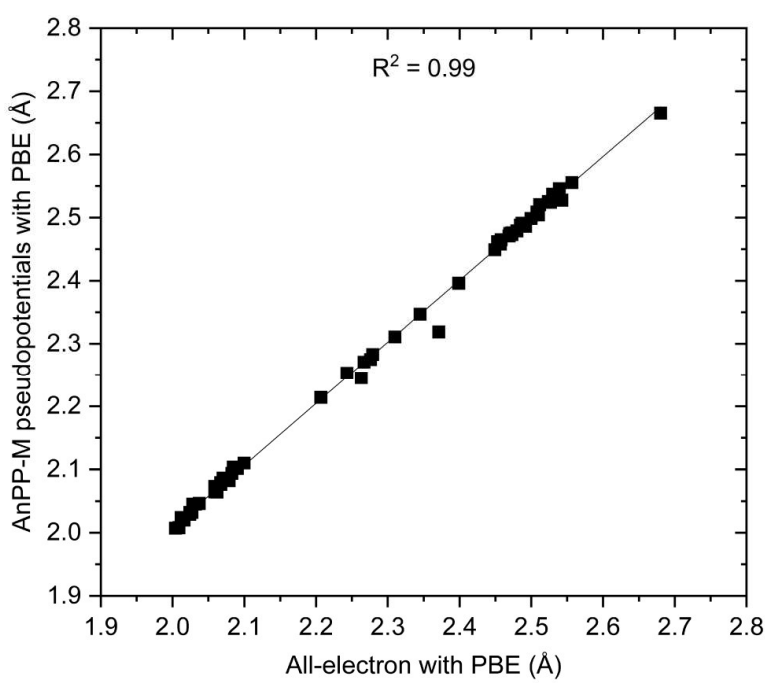

(a)

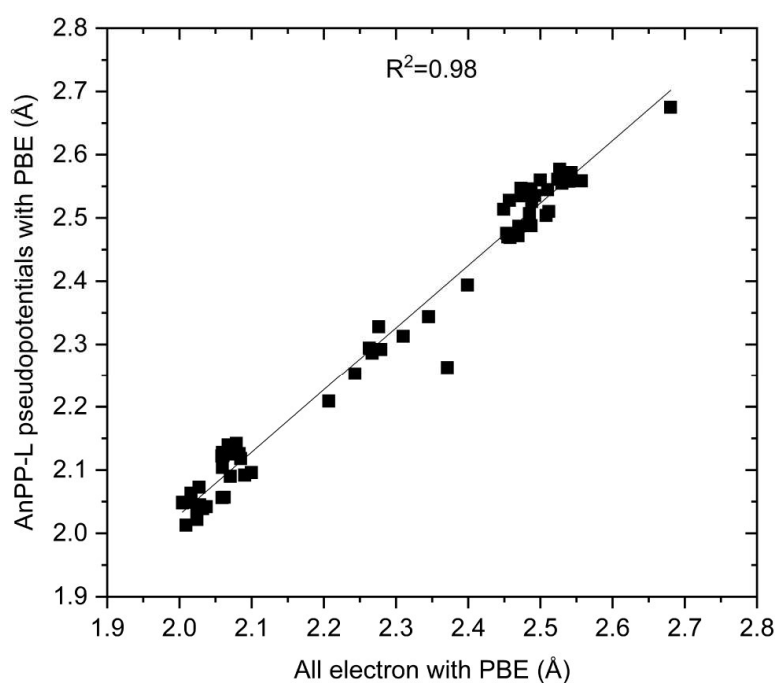

(b)

Figure 8. Comparison of bond lengths of actinide molecules with CP2K using AnPP-M (a) and AnPPL (b) and all-electron calculations using ADF program for compounds in Figure 7. 


\subsubsection{Ligand bond and binding energies}

With the optimized geometries as shown in Figure 7, we calculated the bond energies of actinidehalogen in actinide halides as well as the binding energy between water and actinide ion with III oxidation state in actinide monohydrate. The $\mathrm{An}-\mathrm{X}(\mathrm{X}=\mathrm{F}, \mathrm{Cl})$ bond energies were computed as:

$$
E_{\text {bond }}=\frac{E(A n)+n \times E(X)-E(A n n)}{n}
$$

in which $n=5,6$ for $\mathrm{AnCl}_{5}, \mathrm{AnF}_{5}$ and $\mathrm{AnCl}_{6}, \mathrm{AnF}_{6}$ and $n=3$ for $\mathrm{AnF}_{3}$ and $\mathrm{AnCl}_{3}$. Here $\mathrm{E}(\mathrm{An})$ and $\mathrm{E}(\mathrm{X})$ denote energies of actinide and halogen atoms at PBE level. The binding energies between $\mathrm{An}^{3+}$ and $\mathrm{H}_{2} \mathrm{O}$ in $\mathrm{An}\left(\mathrm{H}_{2} \mathrm{O}\right)^{3+}$ were computed as:

$$
E_{\text {binding }}=E\left(\mathrm{An}^{3+}\right)+E\left(\mathrm{H}_{2} \mathrm{O}\right)-E\left(\mathrm{An}\left(\mathrm{H}_{2} \mathrm{O}\right)^{3+}\right)
$$

The bond energies and binding energies calculated by AnPP-M and AnPP-L pseudopotential at PBE level are larger than energy-consistent pseudopotentials calculations at Hartree-Fock level. ${ }^{28,73}$ In Table 11 we compare the MAD values of bond energy and binding energy for AnPP-M and AnPP-L. We observe considerably smaller errors with MAD $\sim 2.0 \mathrm{kcal} / \mathrm{mol}$ compared to all-electron calculations when using the AnPP-M pseudopotentials. Tables S29 - S35, in part D of the SI, show the bond energies and binding energies of optimized structures obtained with our AnPP-M and AnPP-L pseudopotentials and basis sets with $\mathrm{CP} 2 \mathrm{~K}$ and all-electrons methods with $\mathrm{ADF}$ in detail. 
Table 11. Computed MAD values of bond energies $(\mathrm{kcal} / \mathrm{mol})$ of $\mathrm{An}-\mathrm{X}(\mathrm{X}=\mathrm{F}, \mathrm{Cl})$ in actinide halides with III, V and VI oxidation states for actinides and binding energies of between $\mathrm{An}$ and $\mathrm{H}_{2} \mathrm{O}$ in actinide monohydrate with III oxidation states for actinides using CP2K with our AnPP-L and AnPP-M pseudopotentials and basis sets with respect to all-electron ADF calculations at the PBE level.

\begin{tabular}{ccc}
\hline & \multicolumn{2}{c}{$\mathrm{MAD}$} \\
\cline { 2 - 3 } & $\Delta \mathrm{E}_{\mathrm{AnPP}-\mathrm{L}-\mathrm{AE}}$ & $\Delta \mathrm{E}_{\mathrm{AnPP}-\mathrm{M}-\mathrm{AE}}$ \\
\hline $\mathrm{AnF}_{3}$ & 5.1 & 2.5 \\
$\mathrm{AnF}_{5}$ & 9.8 & 3.1 \\
$\mathrm{AnF}_{6}$ & 10.6 & 2.9 \\
$\mathrm{An}^{3+}-\mathrm{H}_{2} \mathrm{O}$ & 5.0 & 2.3 \\
$\mathrm{AnCl}_{3}$ & 4.1 & 2.4 \\
$\mathrm{AnCl}_{5}$ & 6.0 & 1.8 \\
$\mathrm{AnCl}_{6}$ & 6.7 & 1.9 \\
\hline
\end{tabular}

\subsubsection{Molecular thermodynamic properties}

We calculated the formation enthalpies of $\mathrm{AnO}_{\mathrm{n}}(\mathrm{n}=1,2,3), \mathrm{AnF}_{\mathrm{n}}(\mathrm{n}=1,2,3,4,6)$, and $\mathrm{AnCl}_{\mathrm{n}}$ $(n=3,4)$ molecules, and compared to experimental data, which is available for several early actinide elements. ${ }^{76-79}$ The results are presented in Table 12. Our CP2K calculations of formation enthalpies are based on periodic conditions with uncharged box and an empirical fit to experiment for $U$ with predictive capability for the other elements, following the same approach as done in our previous work ${ }^{41}$, see details in part D of the SI. All computed enthalpies of formation appear in Tables S36 - S45. For low oxidation state actinide molecules, the AnPP-M and AnPP-L pseudopotentials have similar performance. Except for $\mathrm{AnF}_{4}$ and $\mathrm{AnF}_{6}$ molecules, the formation enthalpies calculated by AnPP-M pseudopotentials are closer to experiment than those calculated with the AnPP-L pseudopotentials for high oxidation state molecules. 
Table 12. Computed MAD (kcal/mol) values for enthalpy of formation using CP2K with our AnPP-L and AnPP-M pseudopotentials and basis sets with respect to experiment.

\begin{tabular}{cccc}
\hline \hline & \multirow{2}{*}{ Exp. } & \multicolumn{2}{c}{ MAD } \\
\cline { 3 - 4 } & & $\Delta \mathrm{H}^{\mathrm{f}}$ AnPP-L - Exp. & $\Delta \mathrm{H}^{\mathrm{f}}$ AnPP-M - Exp. \\
\hline $\mathrm{AnO}$ & -11.5 & 9.4 & 9.9 \\
$\mathrm{AnO}_{2}$ & -112.0 & 18.3 & 13.5 \\
$\mathrm{AnO}_{3}$ & -162.8 & 16.6 & 3.0 \\
$\mathrm{AnF}$ & -19.1 & 14.2 & 13.9 \\
$\mathrm{AnF}_{2}$ & -137.8 & 18.7 & 18.3 \\
$\mathrm{AnF}_{3}$ & -269.1 & 34.1 & 34.6 \\
$\mathrm{AnF}_{4}$ & -386.2 & 16.8 & 19.1 \\
$\mathrm{AnF}_{6}$ & -467.9 & 13.2 & 33.2 \\
$\mathrm{AnCl}_{3}$ & -139.1 & 20.0 & 20.4 \\
$\mathrm{AnCl}_{4}$ & -199.9 & 13.7 & 10.6 \\
\hline
\end{tabular}

\section{CONCLUSIONS}

We present here large- and medium-core GTH-type pseudopotentials optimized for all actinide elements from Ac to Lr, as well as their corresponding MOLOPT basis sets with fully contracted coefficients for $s, p, d, f$ and $g$ orbitals. The pseudopotentials and basis sets perform well in a variety of atomic and molecular benchmarks. For the early actinides (Ac to $\mathrm{Cm}$ ), we find that the large-core pseudopotentials have reasonable accuracy with low oxidation states, the medium-core pseudopotentials are superior for the whole series regardless of oxidation state. Given the high efficiency of the GTH pseudopotentials, we anticipate that these datasets will allow calculations and simulations of actinide chemistry in condensed phase systems such as bulk solids, surfaces, interfaces, and molecular species in solution, especially for those elements where very limited or no available information is available. Further development of pseudopotentials and basis sets of actinides with relativistic spin-orbit coupling effects included will be useful for highly accurate calculations of spectroscopic properties. 


\section{Supporting Information}

Discussions on optimization of pseudopotentials and basis sets are presented in part A of the SI (Tables S1 - S6, Figures S1 - S4). Detailed tables about computational methodology are presented in part B of the SI (Tables S7 - S12). Part C of the SI describes transferability results by using different types of pseudopotentials and basis sets (Tables S13 - S19, Figure S5). Detailed tables and Figures about bond lengths, bond energies, binding energies, and formation of enthalpies of small actinide molecules are presented in part D of the SI (Tables S20 - S45, Figure S6). The references of SI are presented in part E of the SI. The resulting GTH pseudopotentials and basis sets of actinides are presented in part F of the SI.

\section{Acknowledgments}

J.-B.L., C.-Q.X., H.-S.H. and J.L. were supported by the National Natural Science Foundation of China (Nos. 22033005 and 21590792). D.C.C. acknowledges support from the Vice Presidency for Research and Innovation, and the College of Engineering, of the University of Nevada, Reno. R. R. and M.-N. T. acknowledge support from Pacific Northwest National Laboratory (PNNL) directed research and development for the chemistry of molten salt reactors (CheMSR) agile initiative. V.A.G. acknowledges support from the US department of Energy, Office of Science, Office of Basic Energy Sciences, Chemical Sciences, Geosciences and Biosciences Division. PNNL is operated by Battelle for the US Department of Energy under Contract DE-AC05-76RL01830. Calculations were performed at Supercomputers at SUSTech Supercomputer Center, Tsinghua National Laboratory for Information Science and Technology, and at Pacific Northwest National Laboratory Institutional Computing.

\section{References and Notes}

(1) Seaborg, G. T. The chemical and radioactive properties of heavy elements. Chem. Eng. News 1945, 23, 2190-2193.

(2) Seaborg, G. T. Place in periodic system and electronic structure of the heaviest elements. Nucleonics 1949, $5,16-36$.

(3) Fox, A. R.; Bart, S. C.; Meyer, K.; Cummins, C. C. Towards uranium catalysts. Nature 2008, 455, 
341-349.

(4) Li, Y.; Su, J.; Mitchell, E.; Zhang, G.; Li, J. Photocatalysis with visible-light-active uranyl complexes. Sci. China Chem. 2013, 56, 1671-1681.

(5) Lu, J. B.; Ma, X. L.; Wang, J. Q.; Jiang, Y. F.; Li, Y.; Hu, H. S.; Xiao, H.; Li, J. The df-d dative bonding in a uranium-cobalt heterobimetallic complex for efficient nitrogen fixation. Inorg. Chem. 2019, 58, 7433-7439.

(6) Wang, G.; Batista, E. R.; Yang, P. Excess electrons on reduced $\mathrm{AnO}_{2}(111)$ surfaces $(\mathrm{An}=\mathrm{Th}, \mathrm{U}$, $\mathrm{Pu}$ ) and their impacts on catalytic water splitting. J. Phys. Chem. C 2019, 123, 30245-30251.

(7) Rinehart, J. D.; Long, J. R. Slow magnetic relaxation in a trigonal prismatic uranium (III) complex. J. Am. Chem. Soc. 2009, 131, 12558-12559.

(8) Mills, D. P.; Moro, F.; McMaster, J.; van Slageren, J.; Lewis, W.; Blake, A. J.; Liddle, S. T. A delocalized arene-bridged diuranium single-molecule magnet. Nat. Chem. 2011, 3, 454-60.

(9) Mougel, V.; Chatelain, L.; Pecaut, J.; Caciuffo, R.; Colineau, E.; Griveau, J. C.; Mazzanti, M. Uranium and manganese assembled in a wheel-shaped nanoscale single-molecule magnet with high spin-reversal barrier. Nat. Chem. 2012, 4, 1011-1017.

(10) Gaggioli, C. A.; Gagliardi, L. Theoretical investigation of plutonium-based single-molecule magnets. Inorg. Chem. 2018, 57, 8098-8105.

(11) Wang, X.; Wang, Y.; Dai, X.; Silver, M. A.; Liu, W.; Li, Y.; Bai, Z.; Gui, D.; Chen, L.; Diwu, J.; Zhou, R.; Chai, Z.; Wang, S. Phase transition triggered aggregation-induced emission in a photoluminescent uranyl-organic framework. Chem. Commun. 2018, 54, 627-630.

(12) Aoki, D.; Huxley, A.; Ressouche, E.; Braithwaite, D.; Flouquet, J.; Brison, J.-P.; Lhotel, E.; Paulsent, C. Coexistence of superconductivity and ferromagnetism in URhGe. Nature 2001, 413, 613-616.

(13) Huy, N. T.; Gasparini, A.; de Nijs, D. E.; Huang, Y.; Klaasse, J. C. P.; Gortenmulder, T.; de Visser, A.; Hamann, A.; Görlach, T.; Löhneysen, H. v. Superconductivity on the border of weak itinerant ferromagnetism in UCoGe. Phys. Rev. Lett. 2007, 99, 067006.

(14) Saxena, S. S.; Agarval, P.; Ahilan, K.; Grosche, F. M.; Haselwimmer, R. K. W.; Steiner, M. J.; Pugh, E.; Walker, I. R.; Julian, S. R.; Monthoux, P.; Lonzarich, G. G.; Huxley, A.; Sheikin, I.; Braithwaite, D.; Flouquet, J. Superconductivity on the border of intinerant-electron ferromagnetism in $\mathrm{UGe}_{2}$. Nature 2000, 406, 587-592. 
(15) Roos, B. O.; Lindh, R.; Malmqvist, P.-Å.; Veryazov, V.; Widmark, P.-O. New relativistic ANO basis sets for actinide atoms. Chem. Phys. Lett. 2005, 409, 295-299.

(16) Pantazis, D. A.; Neese, F. All-Electron Scalar Relativistic Basis Sets for the Actinides. J. Chem. Theory Comput. 2011, 7, 677-684.

(17) Peterson, K. A. Correlation consistent basis sets for actinides. I. The Th and U atoms. J. Chem. Phys. 2015, 142, 074105.

(18) Feng, R.; Peterson, K. A. Correlation consistent basis sets for actinides. II. The atoms Ac and NpLr. J. Chem. Phys. 2017, 147, 084108.

(19) Martins, L. S.; Jorge, F. E.; Franco, M. L.; Ferreira, I. B. All-electron Gaussian basis sets of double zeta quality for the actinides. J. Chem. Phys. 2016, 145, 244113.

(20) de Oliveira, A. Z.; Campos, C. T.; Jorge, F. E.; Ferreira, I. B.; Fantin, P. A. All-electron triple zeta basis sets for the actinides. Comput. Theor. Chem. 2018, 1135, 28-33.

(21) Wadt, W. R.; Hay, P. J. Ab initio studies of the electronic structure and geometry of UF 5 using relativistic effective core potential. J. Am. Chem. Soc. 1979, 101, 5198-5206.

(22) Wadt, W. R. Why $\mathrm{UO}_{2}{ }^{2+}$ is linear and isoelectronic $\mathrm{ThO}_{2}$ is bent. J. Am. Chem. Soc. 1981, 103, 6053-6057.

(23) Wadt, W. R. An ab initio study of low-lying $5 \mathrm{f} \rightarrow 5 \mathrm{f}$ excitations in $\mathrm{PuF}_{6}$. J. Chem. Phys. 1987, 86, 339-346.

(24) VandeVondele, J.; Krack, M.; Mohamed, F.; Parrinello, M.; Chassaing, T.; Hutter, J. Quickstep: Fast and accurate density functional calculations using a mixed Gaussian and plane waves approach. Comput. Phys. Commun. 2005, 167, 103-128.

(25) Ermler, W. C.; Ross, R. B.; Christiansen, P. A. Ab initio relativistic effective potentials with spinorbit operators. VI. Fr through Pu. Int. J. Quantum Chem. 1991, 40, 829-846.

(26) Küchle, W. 1993, Diplomarbeit.

(27) Küchle, W.; Dolg, M.; Stoll, H.; Preuss, H. Energy-adjusted pseudopotentials for the actinides. Parameter sets and test calculations for thorium and thorium monoxide. J. Chem. Phys. 1994, 100, $7535-7542$.

(28) Moritz, A.; Dolg, M. Quasirelativistic energy-consistent 5f-in-core pseudopotentials for pentavalent and hexavalent actinide elements. Theor. Chem. Acc. 2008, 121, 297-306.

(29) Dolg, M.; Cao, X. Accurate relativistic small-core pseudopotentials for actinides. Energy 
adjustment for uranium and first applications to uranium hydride. J. Phys. Chem. A 2009, 113, 12573-12581.

(30) Weigand, A.; Cao, X.; Hangele, T.; Dolg, M. Relativistic small-core pseudopotentials for actinium, thorium, and protactinium. J. Phys. Chem. A 2014, 118, 2519-2530.

(31) Bühl, M.; Kabrede, H. Mechanism of water exchange in aqueous uranyl (VI) ion. A density functional molecular dynamics study. Inorg. Chem. 2006, 45, 3834-3836.

(32) Odoh, S. O.; Bylaska, E. J.; deJong, W. A. Coordination and hydrolysis of plutonium ions in aqueous solution using Car-Parrinello molecular dynamics free energy simulations. J. Phys. Chem. A 2013, 117, 12256-12267.

(33) Rabone, J.; Krack, M. A procedure for bypassing metastable states in local basis set DFT+U calculations and its application to uranium dioxide surfaces. Computational Materials Science 2013, 71, 157-164.

(34) Smirnov, G. S.; Pisarev, V. V.; Stegailov, V. V. Pseudopotential for ab initio calculations of uranium compounds. Journal of Physics: Conf. Series 2018, 946, 012095.

(35) Kleinman, L.; Bylander, D. M. Efficacious form for model pseudopotentials. Phys. Rev. Lett. 1982, $48,1425-1428$.

(36) Goedecker, S.; Teter, S.; Hutter, J. Seperable dual-space Gaussian pseudopotentials. Phys. Rev. B: Condens. Matter Mater. Phys. 1996, 54, 1703-1710.

(37) Hartwigsen, C.; Goedecker, S.; Hutter, J. Relativistic separable dual-space Gaussian pseudopotentials from H to Rn. Phys. Rev. B: Condens. Matter Mater. Phys. 1998, 58, 3641-3662.

(38) Krack, M. Pseudopotentials for $\mathrm{H}$ to $\mathrm{Kr}$ optimized for gradient-corrected exchange-correlation functionals. Theor. Chem. Acc. 2005, 114, 145-152.

(39) Lippert, G.; Hutter, J.; Parrinello, M. A hybrid Gaussian and plane wave density functional scheme. Mol. Phys. 1997, 92, 477-488.

(40) Wang, Y.-G.; Mei, D.; Li, J.; Rousseau, R. DFT+U study on the localized electronic states and their potential role during $\mathrm{H}_{2} \mathrm{O}$ dissociation and $\mathrm{CO}$ oxidation processes on $\mathrm{CeO}_{2}(111)$ Surface. J. Phys. Chem. C 2013, 117, 23082-23089.

(41) Lu, J.-B.; Cantu, D. C.; Nguyen, M. T.; Li, J.; Glezakou, V. A.; Rousseau, R. Norm-Conserving pseudopotentials and basis sets to explore lanthanide chemistry in complex environments. J. Chem. Theory Comput. 2019, 15, 5987-5997. 
(42) VandeVondele, J.; Hutter, J. Gaussian basis sets for accurate calculations on molecular systems in gas and condensed phases. J. Chem. Phys. 2007, 127, 114105.

(43) Hutter, J.; Iannuzzi, M.; Schiffmann, F.; VandeVondele, J. CP2K: atomistic simulations of condensed matter systems. Wiley Interdisciplinary Reviews: Computational Molecular Science 2014, 4, 15-25.

(44) Perdew, J. P.; Burke, K.; Ernzerhof, M. Generalized gradient approximation made simple. Phys. Rev. Lett. 1996, 77, 3865-3868.

(45) Bachelet, G. B.; Hamann, D. R.; Schlüter, M. Pseudopotentials that work: From H to Pu. Phys. Rev. B: Condens. Matter Mater. Phys. 1982, 26, 4199-4228.

(46) Douglas, M.; Kroll, N. M. Quantum electrodynamical corrections to the fine structure of helium. Ann. Phys. 1974, 82, 89-155.

(47) Hess, B. A. Relativistic electronic-structure calculations employing a two-component no-pair formalism with external-field projection operators. Phys. Rev. A: At. Mol. Opt. Phys. 1986, 33, $3742-3748$.

(48) Jansen, G.; Hess, B. A. Revision of the Douglas-Kroll transformation. Phys. Rev. A: At. Mol. Opt. Phys. 1989, 39, 6016-6017.

(49) Wolf, A.; Reiher, M. Hess, B. A. The generalized Douglas-Kroll transformation. J. Chem. Phys. 2002, $117,9215-9226$.

(50) Cotton, S. Lanthanide and actinide chemistry; John Wiley \& Sons: 2013.

(51) $A D F$, version 2016.106; SCM: 2016. http://www.scm.com.

(52) Grimmel, S.; Schoendorff, G.; Wilson, A. K. Gauging the performance of density functionals for lanthanide-containing molecules. J. Chem. Theory Comput. 2016, 12, 1259-1266.

(53) Frisch, M. J. T., G. W.; Schlegel, H. B.; Scuseria, G. E.; Robb, M. A.; Cheeseman, J. R.; Scalmani, G.; Barone, V.; Mennucci, B.; Petersson, G. A.; Nakatsuji, H.; Caricato, M.; Li, X.; Hratchian, H. P.; Izmaylov, A. F.; Bloino, J.; Zheng, G.; Sonnenberg, J. L.; Hada, M.; Ehara, M.; Toyota, K.; Fukuda, R.; Hasegawa, J.; Ishida, M.; Nakajima, T.; Honda, Y.; Kitao, O.; Nakai, H.; Vreven, T.; Montgomery, J. A., Jr.; Peralta, J. E.; Ogliaro, F.; Bearpark, M.; Heyd, J. J.; Brothers, E.; Kudin, K. N.; Staroverov, V. N.; Kobayashi, R.; Normand, J.; Raghavachari, K.; Rendell, A.; Burant, J. C.; Iyengar, S. S.; Tomasi, J.; Cossi, M.; Rega, N.; Millam, N. J.; Klene, M.; Knox, J. E.; Cross, J. B.; Bakken, V.; Adamo, C.; Jaramillo, J.; Gomperts, R.; Stratmann, R. E.; Yazyev, O.; Austin, A. 
J.; Cammi, R.; Pomelli, C.; Ochterski, J. W.; Martin, R. L.; Morokuma, K.; Zakrzewski, V. G.; Voth, G. A.; Salvador, P.; Dannenberg, J. J.; Dapprich, S.; Daniels, A. D.; Farkas, Ö.; Foresman, J. B.; Ortiz, J. V.; Cioslowski, J.; Fox, D. J., Gaussian 09; Gaussian, Inc.: Walingford, CT, 2009.

(54) Cao, X.; Dolg, M.; Stoll, H. Valence basis sets for relativistic energy-consistent small-core actinide pseudopotentials. J. Chem. Phys. 2003, 118, 487-496.

(55) Cao, X.; Dolg, M. Segmented contraction scheme for small-core actinide pseudopotential basis sets. J. Molec. Struct. (Theochem) 2004, 673, 203-209.

(56) Woon, D. E.; Dunning, T. H. Gaussian basis sets for use in correlated molecular calculations. III. The atoms aluminum through argon. J. Chem. Phys. 1993, 98, 1358-1371.

(57) van Lenthe, E.; Baerends, E. J. Optimized slater-type basis sets for elements 1-118. J. Comput. Chem. 2003, 24, 1142-1156.

(58) ADF STO basis set, https://www.scm.com/zorabasis/Welcome.html.

(59)van Lenthe, E.; Baerends, E. J.; Snijders, J. G. Relativistic total energy using regular approximation. J. Chem. Phys. 1994, 101, 9783-9792.

(60) van Lenthe, E.; Snijders, J. G.; Baerends, E. J. The zero-order regular approximation for relativistic effects: The effect of spin-orbit coupling in closed shell molecules. J. Chem. Phys. 1996, 105, $6505-6516$.

(61) van Lenthe, E.; van Leeuwen, R.; Baerends, E. J.; Snijders, J. G. Relativistic regular twocomponent Hamiltonians. Int. J. Quantum Chem. 1996, 57, 281-293.

(62) van Lenthe, E.; Ehlers, A.; Baerends, E. J. Geometry optimizations in the zero-order regular approximation for relativistic effects. J. Chem. Phys. 1999, 110, 8943-8953.

(63) Denning, R. G. Electronic structure and bonding in actinyl ions. Struct. Bonding (Berlin) 1992, 79, 215-276.

(64) Denning, R. G. Electronic structure and bonding in actinyl ion and their analogs. J. Phys. Chem. A 2007, 111, 4125-4143.

(65) Liu, J.-B.; Chen, G. P.; Huang, W.; Clark, D. L.; Schwarz, W. H. E.; Li, J. Bonding trends across the series of tricarbonato-actinyl anions $\left[\left(\mathrm{AnO}_{2}\right)\left(\mathrm{CO}_{3}\right)_{3}\right]^{4-}(\mathrm{An}=\mathrm{U}-\mathrm{Cm})$. Dalton Trans. 2017, 46, $2542-2550$.

(66) Huang, W.; Xu, W.-H.; Schwarz, W. H. E.; Li, J. On the Highest oxidation states of metal elements in $\mathrm{MO}_{4}$ molecules $(\mathrm{M}=\mathrm{Fe}, \mathrm{Ru}, \mathrm{Os}, \mathrm{Hs}, \mathrm{Sm}$, and $\mathrm{Pu})$. Inorg. Chem. 2016, 55, 4616-4625. 
(67) Desclaux, J. P. Relativistic Dirac-Fock expectation values for atoms with $\mathrm{Z}=1$ to $\mathrm{Z}=120$. At. Data Nucl. Data Tables 1973, 12, 311-406.

(68) Zhang, Q.; Hu, S. X.; Qu, H.; Su, J.; Wang, G.; Lu, J. B.; Chen, M.; Zhou, M.; Li, J., Pentavalent Lanthanide Compounds: Formation and Characterization of Praseodymium(V) Oxides. Angew. Chem. Int. Ed. 2016, 55, 6896-900.

(69) Hu, S. X.; Jian, J.; Su, J.; Wu, X.; Li, J.; Zhou, M. Pentavalent lanthanide nitride-oxides: NPrO and $\mathrm{NPrO}^{-}$complexes with N identical with Pr triple bonds. Chem. Sci. 2017, 8, 4035-4043.

(70) Rice, N. T.; Popov, I. A.; Russo, D. R.; Bacsa, J.; Batista, E. R.; Yang, P.; Telser, J.; La Pierre, H. S. Design, Isolation, and Spectroscopic Analysis of a Tetravalent Terbium Complex. J. Am. Chem. Soc. 2019, 141, 13222-13233.

(71) Kramida, A.; Ralchenko, Y.; Reader J. NIST Atomic Spectra Database; National Institute of Standards and Technology: 2014.

(72) Jones, L. H.; Ekberg, S. Potential constants and structure of the UF 5 monomer. J. Chem. Phys. 1977, 67, 2591-2595.

(73) Moritz, A.; Cao, X.; Dolg, M. Quasirelativistic energy-consistent 5f-in-core pseudopotentials for trivalent actinide elements. Theor. Chem. Acc. 2006, 117, 473-481.

(74) Wang, S. G.; Schwarz, W. H. E. Lanthanide diatomics and lanthanide contraction. J. Chem. Phys. 1995, 99, 11687-11695.

(75) Dolg, M.; Stoll, H. Electronic structure calculations for molecules containing lanthanide atoms. Handbook on the physics and chemistry of rare earths (Elsevier) 1996, 22, 607-729.

(76) Konings, R. J. M.; Beneš, O.; Kovács, A.; Manara, D.; Sedmidubský, D.; Gorokhov, L.; Iorish, V. S.; Yungman, V.; Shenyavskaya, E.; Osina, E. The Thermodynamic properties of the f-elements and their compounds. Part 2. The lanthanide and actinide oxides. J. Phys. Chem. Ref. Data 2014, $43,013101$.

(77) OECD, Nuclear Energy Agency, Update on the Chemical thermodynamics of uranium, neptunium, plutonium, americium and technetium, 2003.

(78) OECD, Nuclear Energy Agency, Chemical thermodynamics of neptunium and plutonium, 2000.

(79) OECD, Nuclear Energy Agency, Chemical thermodynamics of thorium, 2008. 
TOC Graphic

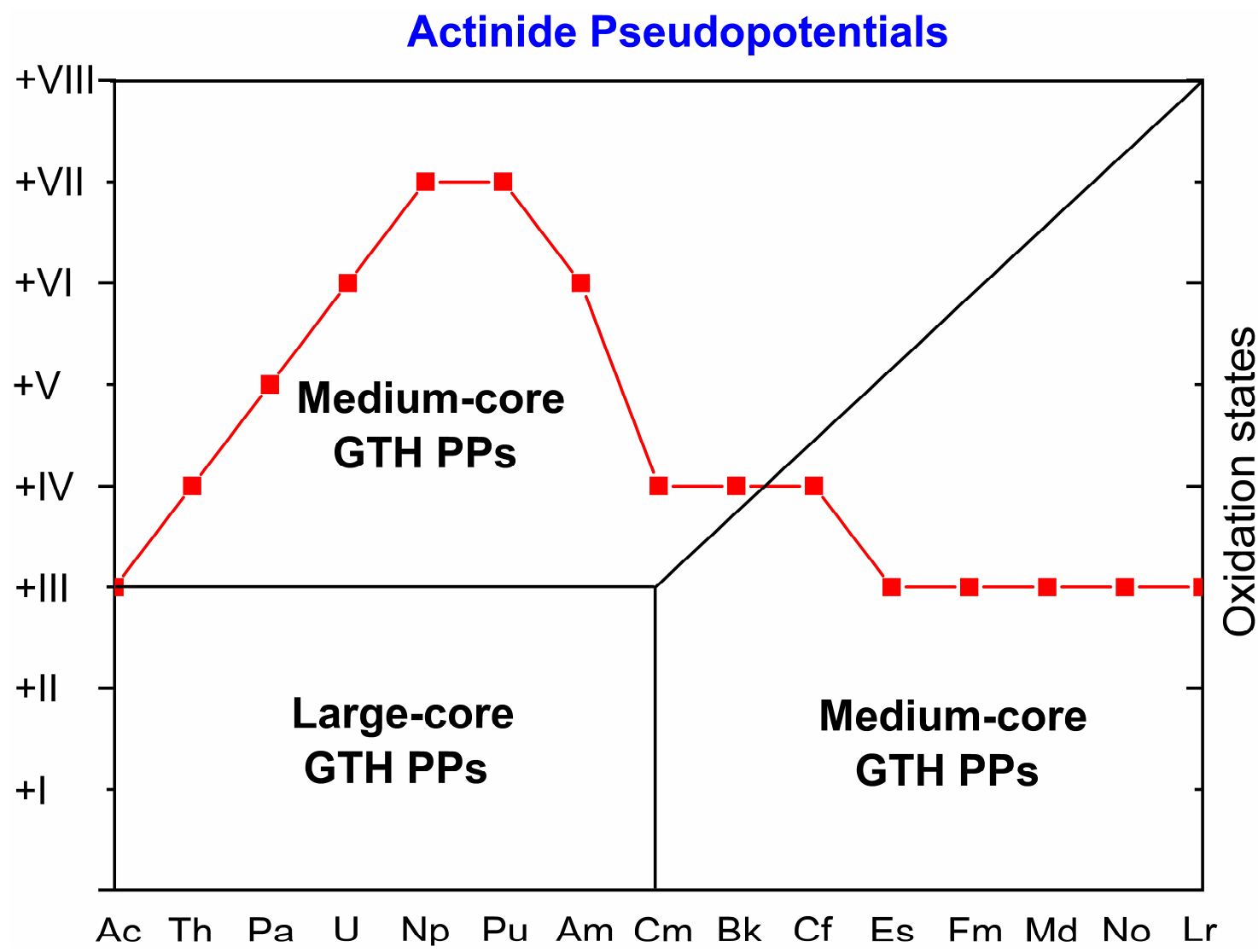

